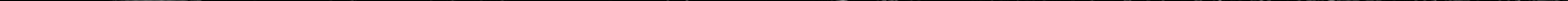




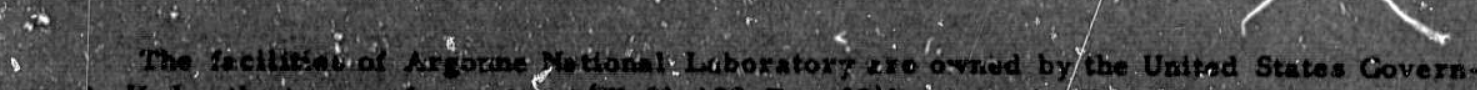

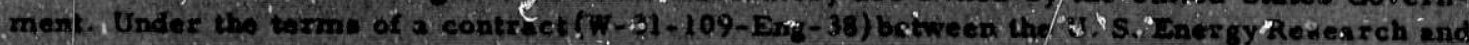

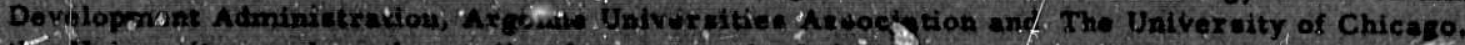

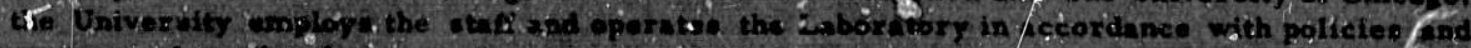

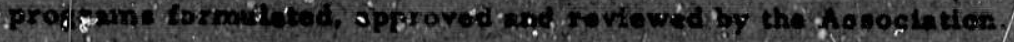

(9)

\section{4}

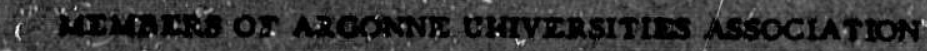

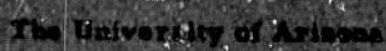

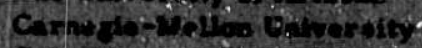

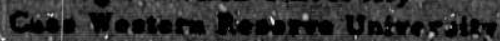

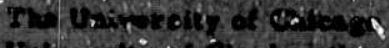

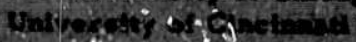

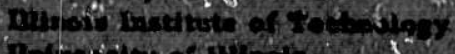

Batrobly as whenth

Yousto phowoty

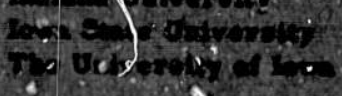

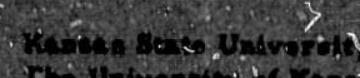

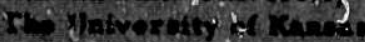

\section{Co volughity}

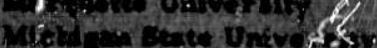

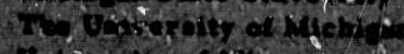

vationk of thing

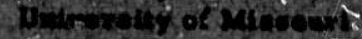

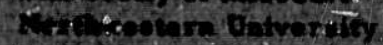

$-\frac{10}{4}$

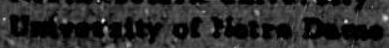

The Ohto state Univarelty

Osio Unveraity

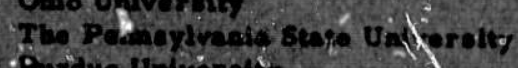

Purdue Ualvarals?

Southith Afichor Univeraity

The onf opolty of Tower at dusth

Whant to Uetwerotey

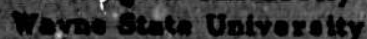

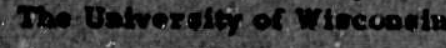

$$
b_{i 2}
$$
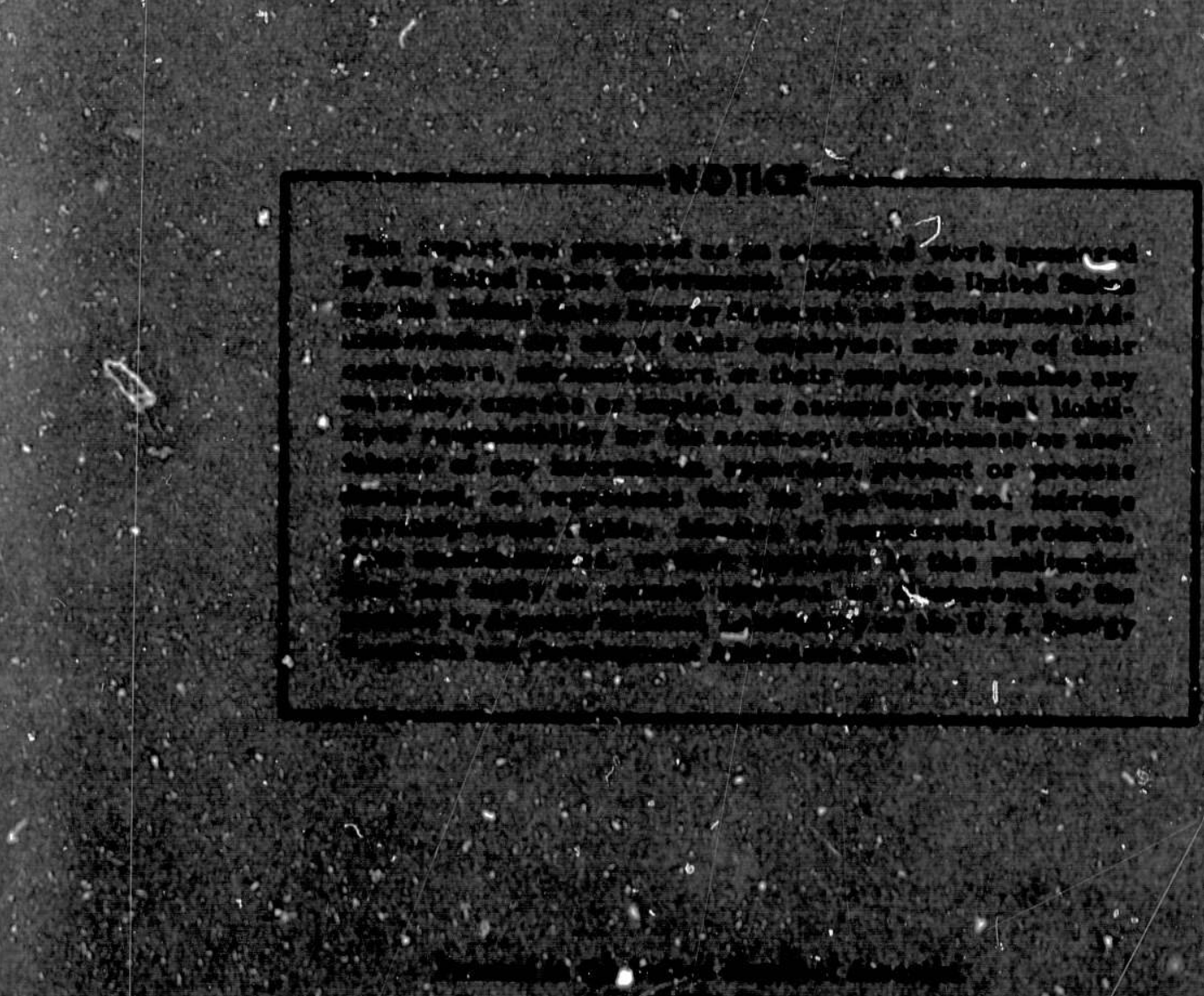

$\omega^{*}$ 4.

(3)
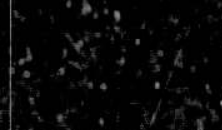
Distribition Category:

Physics-Atomic and Molecular (UC-34a)

ANL $\quad 76-80$

\section{ARGONNE NATIONAL LABORATORY \\ 9700 South Cass Avenue \\ Argonne, Illinols 60439}

CN THE THEORY OF IONIZATION BY ELECTRON COLLISIONS

by

U. Fano*

Department of Physics

The University of Chicago

and

Mitlo Inokuti

Radiological and Enviranmerital Research Division Argonne Nationai Laboratory

June 1976

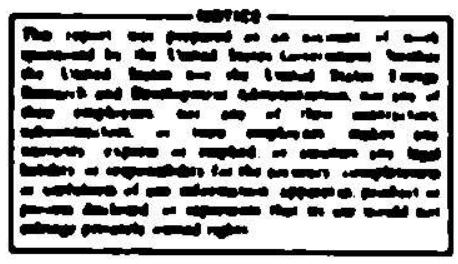

-Work performed in part through Contract No. CO0-1674-119. Appolntee, Faculty Research Part!clpatlon Program, Center for Educational Affatrs, ANL. 


\section{TABLE Of CONTENTS}

Abstrac:

1. Introduction

2. The Seaton-Platzman Plot of $\mathrm{T \sigma}_{\mathrm{f}}$

3. Structure of the Collision Strength $\Omega_{1 f}$

4. Large Impact Parameters 10

5. The Main Problem 13

6. Distorted-Wave Approximation ar.d Its Variants 18

6.1 Partial-Wave Treatment 18

6.2 Treatment in the Momentum Representation 21

6.3 Additional Remarks 26

7. Inner-Shell Ionization 28

8. Peripheral Remarks 30

Raferences 31

Appendix: Note on Extending Quantum Defect Theory to Multiple
Excitations 


\title{
ON THE THEORY OF IONIZATION BY ELECTRON COLLISIONS
}

\author{
U. Fano and Mituo Inokut!
}

The problem of calculating comprehensive seis of cross sections for lonization of atoms and molecules is reviewed. We emphasize targets that are already lonized and lower-energy collisions for which the incident electron must bo treated on a par with target electrons. The physical circumstances are discussed in which porturbation methods should be adequate, and special investgations are recommended to identsfy the relevant ranges of parameters more precisely and dependably. A new R-matrix approach is outlined which should pormlt rather simple, approximate, but dependable calculations when the perturbation mothods fall. 


\section{Introduction}

Cross sections for inelastic collisions of fast electrons have long been accessible to rather dependable calculation by the (first) Born approximation. This approximation falls at lower energies, notably in the range of the crosssection maxima and nearer the threshold. Altemative methods of calculation have been applied in this rarge, but with only limited success, owing, we thini, to our poor understanding of the basic features of the low-energy collisions.

[For example, it is often difficult to disentangle effects of two kinds of approxImations: schematization of the fiamiltonion (such as the use of a phenomenological potential) on the one hand, and an analytical or numerical approximation of its solution on the other.] However, our qualitative understanding has recently progressed considerably, to a point where we may attempt at least a sketchy survey of the whole problem. Our immediate objective is to point out the areas where one can procead confidently by current techniques and those where further development work seems in order.

In low-energy collisions, the incident and target electruns intermingle to such an extent that we feel it necessary to treat them as a single manyelectron complex, except for the cases of high incident angular momenta (as ciscussed in Sec. 4) in which the incident electron fails to penetrate the target. An ionizing collision is then represented by a state of the continuous spectrum of this complex, which evolves from an initial channel with a single electron outside the target to a final channel with two detached electrons.

Detachment of two electrons from a complex has been poorly studied thus far. Accordingly, currerit computing methods (e.g., described by Burke and Seaton 19\%:: Seator 1975; and Burke and Robb 1975) are Inherentlv Incapat), of calculating the probability of this trensition from the initial channel to the final channel under general conditions. However, an $R$-mat-lx approach should permit one to characterize the critjcal central state cf the transition by an effort comparable to - few-configuration SCF calculation of discrete spectral levels. It is suggested in Sec. 5 that such calculations alone might be adequate to estimate total lonization cross sections to about 10 to $20 \%$, and that they could be programmed (at this lovel of accuracy) with a rather modest effort. When the 
Initial arget is itsclf an ion of sufficiently high charge, the transition probability shoulu be amenable to evaluation by a first-order perturbation theory (Sec. 6).

We shall treat collisions of electrons with both neutral atoms and ionized atoms, with considerable emphasis on the latter. However, most of our considera:ions will apply to molecules and to molecular jons as well.

A comprehensive framework for our discussion is provided by crosssection formulas employed extensively by Seaton $(1953,1975)$ and also from a complementary point of view, by Platzman's school (Fano 1975). These formulas represent cross sections as sums of numerous terms (as seen, for example, in Eq. 3.1), and we shall map out which of the terms can be calculated appropriately by various inethods for various ranges of incident energy, types of transitions, or properties of target spectes. The formulation also lends itself to application of sum rules ranging from familiar ones to others whose existence is merely suspected at this time.

2. The Seaton-Platzman Plot of $T \sigma_{f}$

The dimensional element of a wave-mechanical cross section formula is the squared wavelength, $x^{2}$, of the ralative motion of the reactants; in our case, the $x^{2}$ of the electron incident on an atom. The complete formula for the cross section for excitation of the atom from an initial state $i$ to a final state $f$ is wirtten as

$$
\sigma_{f}=\pi x^{2} \omega_{i}^{-1} \Omega_{i f}
$$

where $\omega_{1}$ is the statistical weight of the initial state of the target. and $\Omega_{\text {if }}$ is a welghted sum of (dimensionless) squared transition matrix elcments. Our main goal is to discuss the values of the numerice' factor $\Omega_{\text {if }}$ called the collision strength (Seatun 1953, 1975).

To concentrate on the energy dependence of this factor, one plots the ratio of $\sigma_{f}$ to the factors $\pi x^{2} \omega_{1}^{-1}$ on the rght-hand side of Eq. 2.1. Recall that $x$ is the reciprocal of the incident wave number $k_{0}$, and, hence, $x^{2}$ is inversely proportional to the incident energy $T$. This energy is convoniontly expressed 
In the Rydberg unit $R=\hbar^{2} / 2 \mathrm{ma}_{0}^{2}=13.506 \mathrm{eV}$ where $a_{\jmath}=5.2913 \times 10^{-9} \mathrm{~cm} \mathrm{is}$ the Bohr radius. The numerical quantity to be studied is thus expressed as

$$
\Omega_{\text {if }}=\omega_{1} \frac{T}{R} \frac{\sigma_{f}}{\pi a_{0}{ }^{2}},
$$

in which both $T$ and $J_{f}$ are referred to their natural units.

The collision strength $\Omega_{\text {if }}$ for ioniz.stion starts from zero at its threshold - a behavior understandable from consideration of tha phase space in the final state. But $\Omega_{1 f}$ for cliscrete excltation may start with a finite value. Thereafter, $\Omega_{1 f}$ generally ises, apar. from localized resonances, particularly for the important oplically allowed transitions to be disrussed belcw. For optically forbidden transitions with no change of spin, the rise of $\Omega_{1 f}$ saturates at a finite value. For tra.zsitions with spin change. $\Omega_{\text {if }}$ first peaks and then declines. The steady rise of $\Omega_{\text {if }}$ for optically allowed transitions is due to the occurrence of dipole interactions (1.e., with transfer of one unit of angular momentum) at impact parameters that increase rapidly with increasing energy T. This type of collision, which may occur with very low probability over a very large cross-sectional area, is treated adequately by Bethe's theory in the Bom approximation (Secs. 4.1 and 4.2 of Inokuts 1971). This theory gives"

$$
\left.\Omega_{\text {If }} \rightarrow 4\left(x_{1 f} / a_{0}\right)^{2} \ln \left(4 c_{1 f} T / R\right), \quad \text { (for large } T\right) \quad(\approx .3)
$$

where $x_{\text {if }}$ is the dipole moment of the transition, and $4 c_{\text {if }}$ is a transitiondependent parameter of order unity. The linear dependence of Eq. 2.3 on $\ln T$ led Platzman to plot this quantity against in $T$, for the purpose of determining whether It does, in fact, become linear, and whether its slope does correspond to a plausible value of the relevant dipole moment for photoexcitation. Care is

When the incident electron has a kinetic snergy excoeding tens of keV, relativistic modifications are necessary (Fano 1954). In the definition of $\Omega_{1}$ (Eq. $2.2)$, one then replaces $T / R$ by $(137.036 \beta)^{2}$, where $\beta$ is the ratio of the electron velocity to the light velocity. At the sqme time, one replaces $\ln \left(4 c_{i f} T / R\right)$ by $\ln \left[c_{1 f} \beta^{2} /\left(1-\beta^{2}\right)\right]-\beta^{2}+\ln \left(4 \times 137.036^{2}\right)=\ln \left[c_{1 f} \beta^{2} /\left(1-\beta^{2}\right)\right]-\beta^{2}+11.2268$. Nevertheless, the same target properties $x_{\text {if }}$ and $c_{1 f}$ continue to characterize the high-energy behavior of $\Omega_{1 f}$. 
required in this analysis bacause the approach of $\Omega_{\text {if }}$ to the asymptotic form. 2.3 is deceptively slow, and often has resulted in an overestimate of the coefficient $4\left(x_{i f} / a_{0}\right)^{2}$. The quantity $c_{\text {if }}$ can be evaluated ngorously in principle if the generalized oscillator strength for the transition $i \rightarrow f$ of the target is known (Inokuti 1971), but is not universally related to the energy $E_{1 f}$ of that transition alone. None of the suggestions made so far in the literature about a simple relation of $c_{\text {if }}$ to $E_{\text {if }}$ apparently has a firm theoretical basis.

Whereas the direct plot of $\sigma_{f}$ against $T$ or $\ln T$ shows a major maximum at a small multiple of the threshold energy, this maximum is obscured in the plots of $\delta_{1 f}$. An important compensating advantage is the ease of interpolation between fragmentary data on $\Omega_{1 f}$. The structure of Eq. 2.2 shows that maxima (or minima) of $\sigma_{f}$ correspond to values of $\Omega_{i f}$ at which the tangent of the plot of $\Omega \Omega_{\text {if }}$ vs. $T$ passes through the origin $(T=0)$. On a plot of $\ln \Omega_{\text {if }} v s . \ln T$, the tangent at these points has slope unity. Three of the cross-section plots are exemplified in Figure 1.

3. Structure of the Collision Strength $\Omega_{1 f}$

The collision strength $\Omega_{1 f}$ is defined (Seaton 1975) as a welghted sum over squared elements of the scattering matrix, :

$$
\Omega_{1 f}=\frac{1}{2} \sum_{L S \pi l_{0} n_{f} l_{1}}(2 L+1)(2 S+1)\left|\left(n_{1} l_{1} l_{0}|b(L, S, \pi, T)| n_{f} l_{f} l_{1}\right)\right|^{2} .
$$

Here $(L, S, \pi)$ are the total orbital, spin, and parity quantum numbers of the combined system, electron plus atom, $\left(n_{1} l_{1}\right)$ indicates the initial atomic state, $l_{0}$ the orbital momentum of the incident electron, and $\left(n_{\mathrm{f}}, l_{\mathrm{f}}, l_{1}\right)$ the correspondIng parameters of the final state. The $n_{f}$ may refer to a discrete final state of the atom or to a finite band of its continuous spectrum; it might also refer to an infinitesimal band of the continuum, but in this event, $\Omega_{\text {if }}$ has the dimension of reciprocal energy much as the differential oscillator strength, $d f / d E$, has for photoabsorption. Equation 3.1 presumes that the atoms under study are elther spherical or randomly oriented, and that the incident electron beam is spin-unpolarized. The factor $\frac{f}{2}$ in Eq. 3.1 is the reciprocal of the statistical 
FIG. 1.--Three plots of the total (gross) Ionlzation cross section $\sigma_{\text {ion }}$ of Ar for electron colliston. The data are taken from de Heer and Jansen (1975), who critically examined various experimental results and arrived at recommended values of $\sigma_{10 n}$ and of other cross sections. The hurizontal axis is common to the three plots, and shows the incident kinetic energy $\mathrm{T}$ in $\mathrm{eV}$ on a logarithmic: scale. In contrast, the vertical axis differs for each plot. In plot (a), $\sigma_{\text {ion }}$ itself in units of $10^{-16} \mathrm{~cm}^{2}$ is shown on a linear scale. One sees the familiar bell-shaped zurve with a maximum at $\mathrm{T} \cong 90 \mathrm{eV}$. In plot (b), the vertical axis represents the collision strength for total ionization $\Omega_{\text {lon }}=(T / R)\left(\sigma_{\text {ion }} / \pi a_{l}{ }^{2}\right)$. the statistical weight $\omega_{1}$ of the initial atomic state being unity in this example. The curve of $\Omega_{1 \text { ion }}$ versus log $T$ gradually rises from zero at threshold, and approaches the straight-line asymptotic behavior in accordance with Eq. 2.2. The asymptotic slope corresponds to a theoretically expected value $\left(x_{10 n} / a_{0}\right)^{2}=3.50[\mathrm{KIm}$ et al. (1973)] within several percent. Finally, plot (c) shows $\Omega_{\text {lon }}$ now on a logarithmic vertica! axis whose scale is the same as the horizontal axis. The curve rapidly ises near threshold, and becomes less and less steef at higher and higher $T$, but never flattens out. The continuous curve must take a tangent making $45^{\circ}$ with elther axis at a point, at which $\sigma_{\text {ion }}$ attains the maximum (at $\mathrm{T} \cong 90 \mathrm{eV}$ in this example). The tangent is indicated by the broken line. 

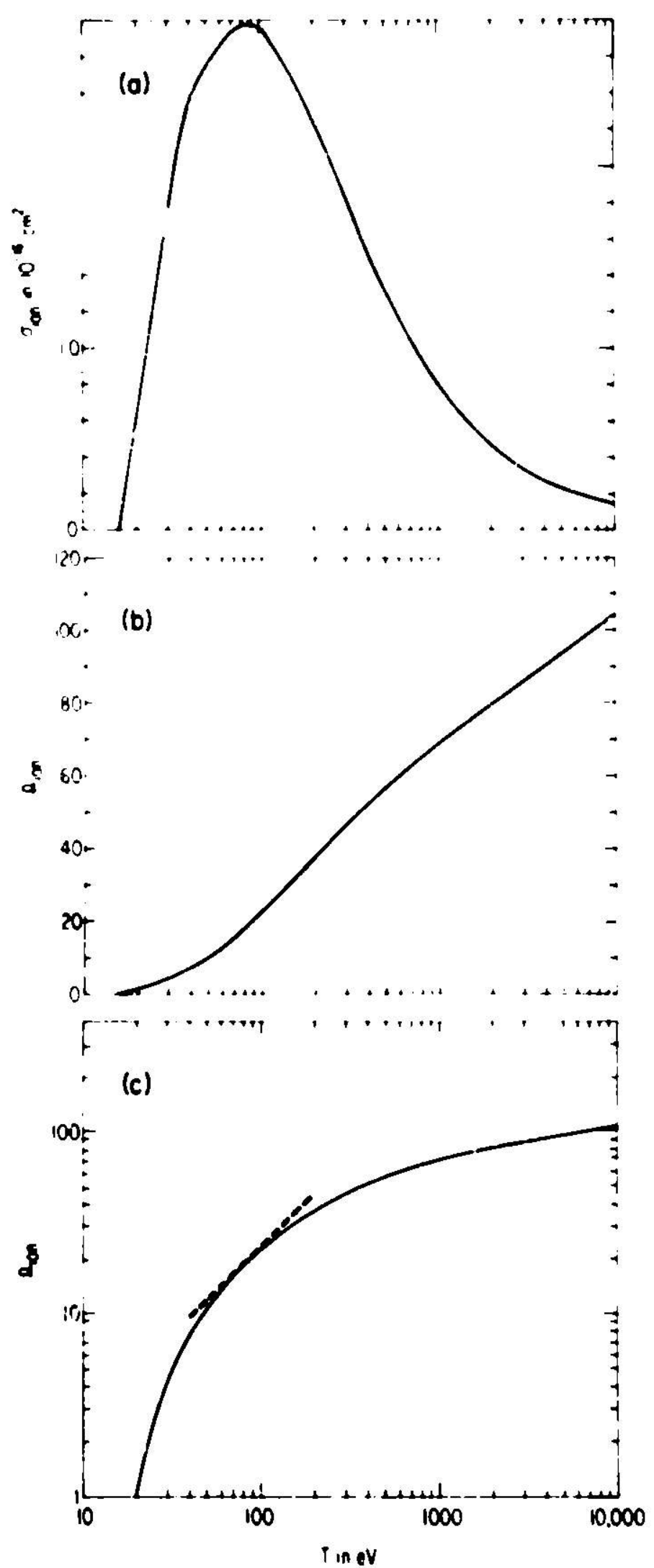

F1B. 1 
weight of the incident electron's spin state.

Equation 3.1 may be supplemented by the insertion of additional quantum numbers as and where needed. For example, Eq. 3.1 implies that the scattering process is independent of spin-orbit coupling. Should this coupling be important and yet so weak that $(L, S)$ remain good quantum numbers, the total momentum $J$ would be added to $(L, S)$, and the weight factor $(2 L+1)(2 S+1)$ replaced by $(2 \mathrm{~J}+1)$. Furthermore, when $(L, S)$ are not conserved in the collision, this pair of indices is replaced by two distinct pairs, $\left(L_{i}, S_{1}\right)$ and $\left(L_{f}, S_{c}\right)$, to be inserted ainong the row and column indices of the $\mathrm{S}$-matrix elements. Note also that the energy of the scattered electron need not appear in Eq. 3.1, being identified by $T$ and $n_{f}$.

Two aspects of tira structire of Eq. 3.1 are central to our remarks:

1) Contributions represented by different terms of the sum may correspond to quite different physical circumstances (e.g., to large or small impact parameters). It may accordingly be appropriate and important to evaluate Jifferent groups of the terms by entirely different methods. Our goal is to discuss which methods are appropriate to various terms.

2) The summation over final-state quantum numbers in Eq. 3.1 arises because we deal with cross sections integrated over certain observabies such as the direction of ejection of atomic electrons. This sum can be extended over an increasing number of indices if one deals with only the combined production of larger blocks of final states. We shall point out how the calculations might be simplified by inquiring only about such larger blocks.

Turning our attention to the magnitude of $\sigma_{f}$, we say that the cross section for an inelastic collision is "small" when the ratio $\sigma_{\mathrm{f}} / \pi \mathrm{a}_{0}^{2}$ in Eq. 2.2 is much smaller than unity. This is usually the case for any collision that raises an atomic electron out of its ground-state shell." (In dealing with excitations of inner-shells or of ions, one may consider the ratio obtained by scaling down $a_{0}$ to the relevant shell radius.) It follows that $\Omega_{1 f}$, or rather $\Omega_{1 f} / \omega_{1}$,

"Much larger cross sections occur, on the other hand, for Intrashell excitations, such as the $5 s^{2} \rightarrow 5 s 5 p$ excitation in $S r$, for which $\sigma_{\text {if }}$ reaches $40 \pi a_{0}{ }^{2}$ at $\mathrm{T}=75 \mathrm{eV}, 1 . e . . \Omega_{\text {If }} \cong 240$ (Chen et al. . 1976). 
is small so long as the energy ratio $T / R$ in $E q .2 .2$ remains neariy unity, but the same does not hold at high energies where Eq. 2.3 may approach an urder of ten. By the same token, we expect individual terms of the sum in Eq. 3.1 to be generally small; the larger value of $\mathrm{Eq} .2 .3$ at high energy derive from cumulative contributions of numerous terms with large values of $\ell_{0}$.

The generally small value of the individual terms of Eq. $3.1 \mathrm{might}$ suggest that their evaluation can proceed by perturbative procedures. (Th1s indication is often verified but should not be taken for granted without detailed analysis.) The first-order-perturbatior, expression of the matrix elements in Eq. 3.1 is (Section $P$ of Wu and Ohmura 1962)

$$
\left(n_{1} \ell_{1} \ell_{0}|\&(L, S, \pi, T)| n_{\ell_{\mathrm{f}} \ell_{1}}\right) \cong 2 \pi i\left(n_{1} \ell_{1} \ell_{0}|V| n_{\mathrm{f}} \ell_{1} \ell_{1}\right),
$$

where $V$ is the in teraction to be discussed below in detail. Perturbative treatments break down certainly when the right-hand side of $\mathrm{Eq} .3 .2$ approaches unity, as was verified, e.g., in early studies of low-energy collisions by Seaton and co-workers (Seaton 1955,1961 and Burke and Seaton 1961). Even very small va'lues of $\mathrm{Eq}$. 3.2 may, however, prove deceptive as in the example of optically forbidden $s \rightarrow d$ excitations for which the main contribution arises from second-order $s \rightarrow p \rightarrow d$ processes (as discussed by Vainshtein and Presnyakov 1968, Korff, Chung, and Lin 1973, for example.) Accordingly, application of Eq. 3.2 must be accompanied by a survey of the whole range of off-diagonal elements of the Hamiltonian.

The interpretation and application of the perturbative treatment require clarfication in several respects.

(a) The two sets of quantum numbers $\left(n_{1} l_{1} l_{0}\right)$ and $\left(n_{f} l_{f} l_{1}\right)$ indicate alternate members of a single orthonormal basis set. This set is so chosen that a major part $\mathrm{H}_{0}$ of the Hamiltonian $\mathrm{H}$ for the combined system (electron plus atom) is diagonal. The "interaction" symbol $\mathrm{V}=\mathrm{H}-\mathrm{H}_{0}$ then denotes whichever part of $H$ that has off-dlagonal elements in the $\mathrm{H}_{0}$-reprosentation. This is essential to a consistent formulation of the perturbation theory in general, as seen, for example, in the theory of atomic spectra. 
(b) Frequently, perturbative treatments of atomic collisions have been formulated differently. (See p. 349 of Mott and Massey 1965 and Sec. 2.8 of Molseiwitsch and Smith 1968.) In the interest of more faithfully characterizing the initial state and the final state (at large distances between the atom and the projectile electron, in particular), many workers have used a member of a basis set for the initial state $\left(n_{1} \ell_{1} l_{0}\right)$ and a member of an entirely different basis set for the final state $\left(n_{\mathrm{f}} \ell_{\mathrm{f}} \boldsymbol{l}_{1}\right)$, thereby ignoring the basic consistency requirement stated in (a). This neglect of the consistency requirement has been most frequently seen in the calculation (as reviewed by Rudge 1968) of ionization cross sections (when $n_{f} l_{f}$ belongs to continua of the target atom). We suspect that the violation of the consistency requirement could have led to serfous errors in resulting cross section evaluations.

(c) Although the interaction V in Eq. 3.1 has the physical dimension of energy, this dimensionality is compensated for exactly by the normalization per unit energy of the incident and scattered electron's wavefunction, which will make the matrix element dimensionless. If, In addition, the final state $\left(n_{\mathrm{f}} \mathbf{l}_{\mathrm{f}}\right)$ of the target atom is ionized and is therefore also normalized per unit energy, the squared matrix element has the dimension of reciprocal energy, in accordance with the discussion of $\Omega_{\text {if }}$ following Eq. 3.1. In this event, any statement as to the smallness of the interaction matrix element must refer to the integral of its square over a finite spectral range of $n_{f}$.

Further discussions on perturbative treatments are presented ir. Sacs. 4 and 6.

\section{Large Impact Parameters}

Particularly simple are the collisions in which the incident electron does not actually traverse the target atom, and hence should not become strongly correlated with the atomic electrons. Insofar as the distance between the Incident and the atomic electrons does nct become very small, their interactions remaln rather weak. Hence, these collisions are prime candidates for dependable application of the Bom approximation. 
The classical concept of large impact parameters should now be adapted for application to our quantum problem. It is loosely assoclated (by a Fourler transformation) to small momentum transfer in the collision. In the frame of Secs. 2 and 3, we associate large impact parameters with large values of the incident orbital momentum $\ell_{0^{\circ}}$ An electron of given $\ell_{0}$ at radial distance $r$ is subject to the centrifugal potential

$$
\ell_{0}\left(\ell_{0}+1\right)\left(a_{0} / r\right)^{2} R \text {; }
$$

If this potential is rauch larger than $T$ when $r$ equals the atomic radius, it will prevent the electron from penetratirg the atom.

To apply this argument quantitatively, consider in the first place how the lack of penetration is represented by the radial wavefunction for angular

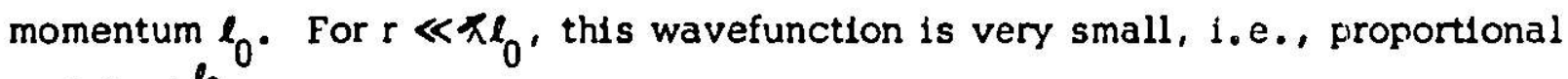
io $\left(r / x_{0}\right)^{l_{0}}$. Then we say that the impact parameter is larger than the atomic radius if the radial overlap of this wavefunction with the initial wavefunction of atomic electrons is negligible for the purposes of any specific application. Negligible overlap implies also negligible exchange effects.

We call $l_{c}$ the smallest value of $l_{0}$ for which the overlap is negligible at any one value of $T$, and assume tentatively that the first-order perturbation formula 3.2 is applicable to ali terms of Eq. 3.1 with $l_{0} \geq l_{c}$ and $l_{1} \geq l_{c}$. (Notice that $l_{c}$ is dependent on $T_{i}$ it is proportional to $T^{\frac{1}{2}}$ and therefore it increases gradually with T.) Application of the first-order treatment in our context means that the interaction operator in $\mathrm{Eq} .3 .2$ is set equal to $\sum_{j} e^{2},\left|\vec{r}-\vec{r}_{j}\right|$, where $\vec{r}$ is the incident electron's position and $\vec{r}_{j}$ that of an atomic electron, and that the incident electron's wavefunctions are taken to be the Riccati-Bessel $j_{l_{0}}\left(k_{0} r\right)$ and $l_{l_{1}}\left(k_{1} r\right)$, where $k_{0}$ is the wave number correspond ing to the enorgy $T$, and $k_{1}$ corresponds to the energy of the scattered electron. A survey study of such applications appears desirable now to cover the followIng points.

a) Estimation of the matrix element (Eq. 3.2) and of its dependence on atomic parameters for typical values of the quantidin numbers;

b) Eistimation of the accuracy of the first-order approxdmation (Eq. 3.2) 
under those conditions;

c) Estimaition of the neglected overlap with atomic wavefunctions for altemative values of the cut-off orbital momentum $\ell_{\mathrm{c}}$.

With the restriction thus imposed (i.e., for $l_{0}>l_{c}$ ), first-order perturbation treatment might well remain valld down to the threshold energy of each process, at least for the optically allowed transitions that give the dominant contribution to $\Omega_{1 f}$. Because very large values of $l_{0}$ may contribute appreciably to $\Omega_{\text {If }}$, It might be expedient to evaluate the $\sum l_{0}>l_{\mathrm{C}}$ in Eq. 3.1 as the difference between two terms: the complete sum evaluated by integration over the momentum transfer (Eq. 6.9) on the one hand, and the contribution of the terms $\sum l_{0} \leq l_{C}$ each evaluated by Eq. 3.2 on the other hand; the subtraction eliminates the error implicit in the calculation of either term.

The summation over $l_{0}$ does not, in practice, extend to infinite $l_{0}$, but is effectively cut off at a maximum value $l_{\max }$ of $l_{0}$. The value of $l_{\max }$ is set at

$$
l_{\max }=b_{\max } k_{0} \text {, }
$$

where $b_{\max }$ is the maximum impact parameter at which the inelastic collision is barely possible. This value $b_{\max }$ is related, by a Fourier analysis, to the minimum momentum transfer consistent with the given energy loss, $E_{1 f}$ $\hbar K_{\min } \cong \mathrm{mE}_{\text {if }} /\left(\hbar \mathrm{k}_{0}\right)$, as

$$
b_{\max } \cong 1 / k_{\min } \text {. }
$$

Therefore, we have an estimate

$$
l_{\max } \cong k_{0} / k_{\min } \cong\left(\hbar k_{0}\right)^{2} /\left(m E_{1 f}\right) \sim 2 T / E_{1 f} .
$$

One may wonder about the error Introduced by evaluating the matrix element (Eq. 3.2) with undistorted Riccati-Bessel wavefunctions as some distortion should arise even for collisions with neutral atoms, not-only from the small overlap with the atom itself, but also from the long-range interaction due to polarization of the target. The distortion is far more important for collision: with lons owing to their Coulomb field. This matter will be consider'sd in Sec. 6 , in the context of the Coulomb-Bom or distorted-wave approxdmation. 
There are indications that such effects, even when appreciable for individual elements of the scattering matrix, may be averaged out to a substantial extent in the eventual summation of Eq. 3.1. Indeed, results of distorted-wave approximations (e.g., Madison and Shelton 1973, Thomas et al. 1974, and Chutjian and Thomas 1975) show that integrated cross sections are far less sensitive to distortion effects than differential cross sections, 1.e., angular distributions of inelastically scattered electrons.

\section{The Main Problem}

When the incident electron penetrates the target atom and its energy does not exceed three or four times the ionization threshold, the electron and target should be treated as a single system in a state of its continuous spectrum. Ionization is then regarded as a transition of this system from a channel with a single detached electron to a channol with two detached electrons. Treatment of this transition by a Coulomb-Bom or related approximation becomes possible only if the system has a rather large net positive charge, as discussed at the end of this section and in the next section. Furthermore, neither the closecoupling method (Burke and Seaton 1971, Seaton 1975) nor the R-matrix method, as currently applied (Burke and Robb 1975, Lee 1974), can deal with ionization by collision, even though they are very effective for calculating cross sections for lower excitations near their threshold. Their main limitation lles in the restriction to escape of a single electron. Also, the calculation of excitations becomes unrealistic at energies above the thresholds for numerous altemative excitation channels or for tonization; only a few of these channels can be included explicitly, and replacement of the others by pseudo-states leaves much to be desired.

The main approach to the problem seems to require ext onding the $R$ matrix method to deal with two olectrons emerging from the atomic-core boundary on which the $R$ matrix is defined. A proliminary study of this approach is included in this report as an Appondix. It outlines a program whose full implementation will requite a great deal of roscarch and development. 
Here we outline how that program might be abbreviated if one asks only for the sum of cross sections for large classes of processes (e.g., for total Ionization) rather than for the separate cross sections of individual processes. An opportunity for simplification is then provided by the fact that the motion of the two electrons proceeds essentially semiclassically at large radial distances without affording elther electron much chance of falling al! the way back into the ionized core. More specifically, the electron correlations that may decide whether the collision leads to ionization, or at least to higher excitadon, take place within a radius of four to elght atomic units from the nucleus. Correlations at longer ranges, on the other hand, produce only minor exchanges of energy between two electrons and thus play a i essential role only for finer features of collision spectra (such as threshuld ionization), which are irrelevant to our present purposes.

The suggested plan is to calculate the $R$ matrix on a boundary surface in the two-electron configuration space. This matrix will provide the probability amplitude that two electrons emerge from a collision initiated by a single Incident electron. The $R$ matrix is to be calculated by the approach of Lee (1974), 1.e., by solving the Schroedinger equation by a Hartree-Fock-Roothaan procedure at any desired energy within the finite volume enclosed by the boundary whose radius should be of the order of $10 \mathrm{a} . \mathrm{u}$. This variational procedure yields the radial wavefunctions of the electrons (including the incident one) in analytic form with numerically determined coefficients. According to the Appendix, the pair of radial variables of the two orbitals that extend farthest from the nucleus should $\mathrm{D}$ s expressed in the hyperspherical polar coordinate form $(R \cos \alpha, R \sin \alpha)$ rather than in its usual form, $\left(r_{1}, r_{2}\right)$. Using these coordinates, one delimits the range of variables within which to solve the Schroedinger equation by setting a maximum value $R_{0}$ to the combined variable $R=\left(r_{1}{ }^{2}+r_{2}{ }^{2}\right)^{\frac{1}{2}}$ instead of setting a maximum value $r_{0}$ for $r_{1}$ and $r_{2}$ separately. Thus a hyperspherical boundary surface $R_{0}$ replaces the more obvious cholce of a spherical surface in ths physical space (see Figure 2).

On a boundary surface itself, the wavefunction depends on the directions $\left(\hat{r}_{1}, \hat{r}_{2}\right)$ of the electrons and on the mock-angle $\alpha$ which represents the ratio of 


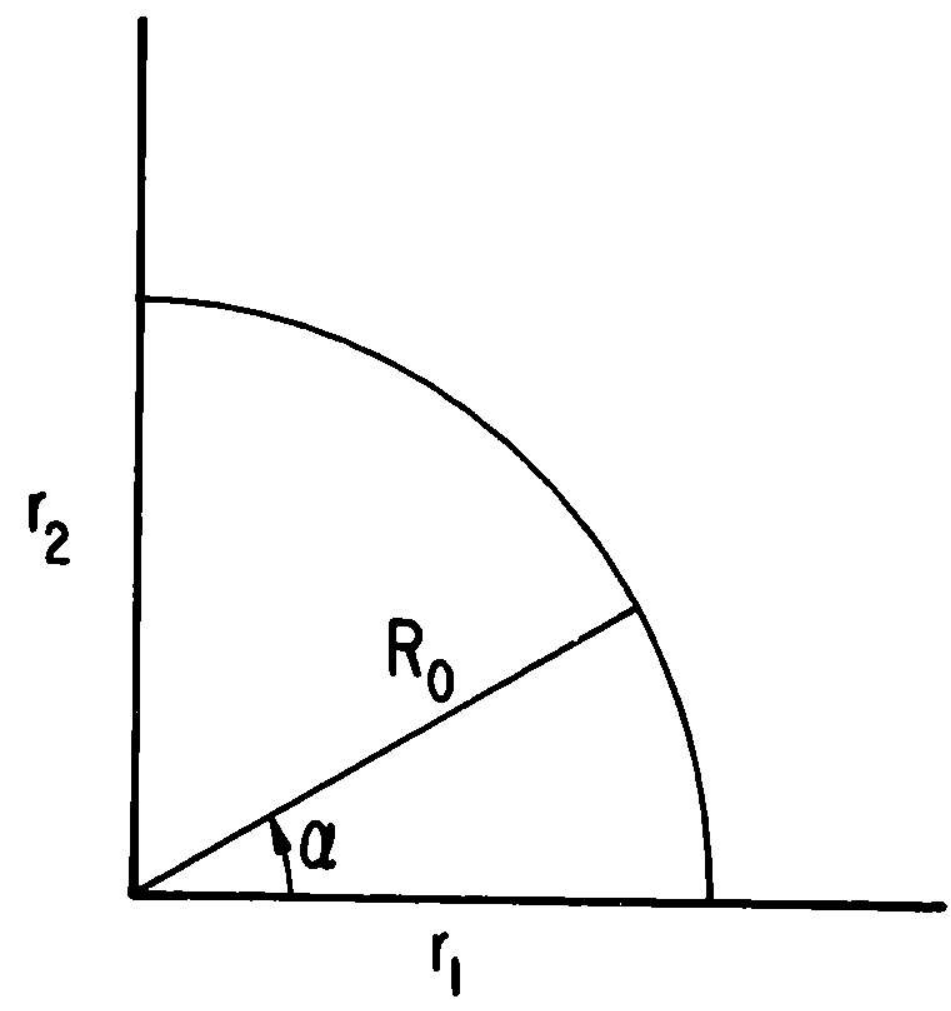

FIG. 2. - - The boundary surface on which the $R$ matrix is to be calculated. The ordinary radial coordinates, $r_{1}$ and $r_{2}$, define the rectangular axes. The hyperspherical polar angle is $\alpha=\tan ^{-1}\left(r_{2} / r_{1}\right)$. The arc represents the intersection of the six-dimensional hypersphere, $R=R_{0}$, with the coordinate plane, $\left(r_{1} r_{2}\right)$. The remaining four dimensions of the configuration space, corresponding to electron radial directions, $\hat{r}_{1}$ and $\hat{r}_{2}$, are not shown.

radial distances of the two escaping electrons from the nucleus. This dependence is expanded into spherical harmonics of $\hat{r}_{1}$ and $\hat{r}_{2}$, corresponding to their altemative orbital momenta, $\ell_{1}$ and $l_{2}$, and into an approprlate set of functions $\mathrm{g}^{\ell_{1} \ell_{2}} \mathrm{~m}\left(\mathrm{R}_{0} ; \alpha\right)$ (discussed in the Appendix) with different numbers of nodes $\mathrm{m}$. The $R$ matrix consists of the coefficients of this expansion for a complete set of collision states. The first (nodeless) function of this set, $g^{l_{1} l_{2}}{ }_{0}\left(R_{0} ; \alpha\right)$. has nonzero values on the boundary $\left(R=R_{0}\right)$ only for extreme values of $\alpha$ (near $0^{\circ}$ or $90^{\circ}$ ), which correspond to small values of $r_{2}=R \sin \alpha$ or of $r_{1}=R \cos \alpha$, and hence to the case in which one of the two electrons actually remains closely attached to the atom; thus it represents the elastic channel of the collision. Successive functions $g^{l_{1} l_{2}}\left(R_{0} ; \alpha\right)$ with $m \geq 1$ should have nonnegligible values throughout the range of $\alpha$, from $0^{\circ}$ to $90^{\circ}$, for a value of $R_{0}$ appropriate to our problem, and should thus represent components of the full wavefunction in which two electrons share the avallable excitation energy so that they can both escape from the target.

A wavefunction of interest should have then the following structure on and near the boundary surface $R=R_{0}$. It should consist firstly of an incident 
wave, progressing toward $\mathrm{R}=0$, and including only angular components with the quantum number $m=0$ (1.e., nodeless in $\alpha)$ and with orbital and spin angular momenta coupled as appropriate. The outgoing wave should consist, in principle, of components in all $\left(m, \ell_{1}, \ell_{2}\right)$ channels accessible from the incident channel with conservation of (L,S, partty); the expansion should also include terms corresponding to all relevant alternative angular momenta of the residual lonized core. The coefficients of the various terms of this outgoingwave expansion constitute elements of the $R$ matrix when properly normalized. Their calculation is achleved by solving the Schroedinger equation within the range $\mathrm{R} \leq \mathrm{R}_{0}$ only.

The point we anticipate here is that, for incident energies $\mathrm{T}$ no larger than a small multiple of the threshold $I$ and for values of $R_{0}$ of the indicated magnitude, the amplitudes of outgoing-wave components with $\mathrm{m}>1$ should be negligible. The entire information of major interest should then be included in the one R-matrix element (or a few) corresponding to excitation from the incident $(m=0)$ to the outgoing inelastic $m=1$ channel(s).

To separate the ionization from the excitation contributions to this total probability, we suggest here that once an atomic electron is excited out of its ground-state shell, its subsequent sharing of energy with the incident electron takes place in the outer region $R>R_{0}$, where a WKB approximation is probably applicable. Under this condition, the wave propagation outside $R_{0}$ would not be diffracted back into the inner zone. Moreover, we surmise that under our conditions, 1.e., with a single inelastic-channel wave propagating outwards, the avallable energy will be partitioned between the two electrons with essentially uniform distribition in the spectrum. A flat distribution of energy in the two.electron spectrum has indeed been derived analytically by Wannior for lonization by collision, albelt in a narrow range of total energy, and numerically over a broad energy range of two-electron photolonization by Chang and Poo (1975).

Thus, we assume that all altemative sharings of energy by the two electrons have substantially equal probability, on an energy scale, and we nood not worry, at this staje, about studying the outer region $R>R_{0}$ in detall. 
In the event of ionization, the secondary-electron's escape energy may range from zero to $\frac{1}{2}(T-I)$, where $T$ is the incident energy and I the ionization threshold. Call $\Delta$ the range of excitation energies from the lowest unoccupied shell to the ionization threstsld, which is $\sim 3-4 \mathrm{eV}$ for a neutral atom. Dur assumption implies that the fraction $F_{I}$ of processes leading to fonization rather than excitation is

$$
F_{I}=\frac{\frac{1}{2}(T-I)}{\frac{1}{2}(T-I)+\Delta}
$$

This estimate may be corrected, if warranted, for minor effects such as the depression in the ionization or excitation ylelds very near the threshold energy. A systematic departure from the assumption underlying Eq. 5.1 appears with increasing $T$, in that excitations and ionlzations with low secondary energles become increasingly more likely than ionlzations with nearly equal partition of energy between the two electrons. The onset of this effect should be marked by non-negligible values of the coefficient of the next eigenfunction $\mathrm{g}^{\ell_{1} \ell_{2}}{ }_{2}\left(R_{0} ; \alpha\right)$ with two nodes; it remains to be studled how $F_{I}$ will vary with $\mathrm{T}$ as the coefficients of successive functions $\mathrm{g}^{\ell_{1} l_{2}} \mathrm{~m}$ increase.

Programming and performing calculatlons of the $R$ matrix according to this plan should require only a modest effort in vien of Lee's (1974) experience with single-electron photoexcitation of Ar. (His idaptation of a bound state program to an R-matrix calculation required two 1.0 three student-months, and the running time for the calculation over an energy range of approximately $5 \mathrm{eV}$ was less than 5 minutes.) For the application to two escaping electrons, the radial functions of these electrons might be cast in a polynomial form, which permits ready application of boundary conditions on the hypersphere $R=R_{0}$ by substituting $r_{1}=R \cos \alpha, r_{2}=R \sin \alpha$.

As noted at the beginning of this section, a first-order perturbation approach is warranted when the target atom 18 inulliply lonized, because the Interactions botween the incident and atomic electrons (and among the atorifc electrons) become woak as compared with the atomic fleld strength in the region of the valence shell. A formal treatment may utillze Layzer's (1959) 
expansion into inverse powers of the net iontc charge $z$. Recent test calculations at Los Alamos (W. D. Robb, personal communtcation) indicate that a Coulomb-Bom perturbation approach becomes adequate for $z>5$. This type of treatment is discussed further in the next section. Here we note that its adequacy could be tested within the framework of an R-matrix calculation by examining whether the interaction matrix elements are sufficiently small to permit carrying it out by a perturbation technique.

\section{Distorted-Wave Approximation and Its Variants}

\subsection{Partial-Wave Treatment}

Following up the final passages of Secs. 3 and 5, we now discuss the first-order perturbation treatments, which are especially appropriate for electron inelastic collisions with highly stripped ions.

It seems essential to us that the incident electron and a target electron (to be excited or lonized) should be treated on equal footing. in particular, both electrons should be subject to the same potentsal $U$ due to the target core, which we may call the distortion potential and which includes relevant exchange terms. At large distances $r, U$ approaches the Coulomb potential $U \rightarrow-2 e^{2} / r$,

where $z$ is the net charge of the target core after lonization, but it becomes stronger than Eq. 6.1 within the core. The motion of each electron (labeled by 1) is described in the zero-order approximation by an eigenstate of $\mathrm{H}_{0}(j)=$ $K(1)+U(j)$, where $K$ represents the kinetic energy. (The mutual screening of the two electrons is probably of minor importance, as noted below.) in the fincl state, the motion of each eluctron is again described by another eigenstate of the same $\mathrm{H}_{0}$. The inelasticity is caused by the residual interaction $V=H-\sum_{j} H_{0}(j)$, where $H 18$ the full Hamiltonian, and the relevant part of $V$ is the electron-electron interaction $\theta^{2} / r_{12}$, which 18 woaker then $U$ in a ratio of about $1 / 2$. Consequently, we regand the inelastic collision as a tratistition from an elgenstate of $\sum_{j} H_{0}(j)$ to anottint, caused by $v$, ard apply the standard Erst-order ferturbation theory to this process. The result is expressed by Eq. 3.2. 
A number of remarks are in order for the precise characterlzation of this calculational scheme and for clarification of its implications. First, we have singled out one of the target electrons for brevity of discussion. Usually there are many electrons even in a particular shell of interest in the target. Indeed, the initial state has one free electron and $\mathrm{N}$ bound electrons, and the final state for Ionization has two free electrons and $\mathrm{N}-1$ bound electrons, where $\mathrm{N}$ is the total number of target electrons. An initial step in the formulation must Include a full determinantal wavefunction for $\mathrm{N}+1$ electrons (or a sultable linear combination of such determinantal wavefunctions as needed to construct an eigenstate of the conserved quantum numbers such as $L, S, J$, and parity). The next step is the reduction to a two-electron problem; in other words, one singles out the two electrons from the rest, 1.e., from $\mathrm{N}-1$ spectator electrons. This reduction is justified because $V$ is a sum of two-electron operators and acts on states of identical electrons. Carrying through this reduction procedure in general is indeed a nontrivial task of angular-momentum algebra, but is routine in atomic spectroscopy.

Seccnd, the procedure described above implies taking account of electron-exchange effects. As a result, the expression for the $\mathrm{S}$ matrix element (Eq. 3.2) includes two classes of terms, one class representing direct scattering, and the other class representing exchange scattering in which the lakeis of the two electrons are interchanged in the final state $n_{f} l_{f_{1}}$, as indicated by $n_{f} \ell_{f} \geqslant \ell_{1}$.

Third, the first.-order treatment of $V$ is justified by considerations of energy scales. We begin with the recognition that the asymptotic form (Eq. 6.1) of $U$ Immediately Implies that the energies of the initlal state and the final state in the $H_{0}$-representation are roughly measured in terms of $z^{2} R$, where $R$ is the Rydberg unit. The strength of the interaction $e^{2} / r_{12}$ is always of the order of unity, but the mean reciprocal distance $\left(1 / r_{12}\right)$ of the two electrons is of the order $z / a_{0}$ in the Important range. Therefore, the ratio of $V$ to $U$ is roughly $1 / 2$, which 18 , by definition, a small quantity for highly stripped lons. (The above scallng is only qualitatuvely true and 18 violated because of non-Coulomble parts of $U$, which are important, as noted below.) 
Fourth, the calculational scheme we have outlined above is a departure from often-practiced distorted-wave calculations. In the traditional approach exemplified on p. 349 of the Mott-Massey textbook (1965), the distorted-wave approximation (DWA) is viewed as a truncation of the close-colpling approximation, which starts out with the presumption that all the eigenstates of the türget atnm or ion are exactly known in advance and that these eigenstates should be the most approprlate basis in which to expand the $\mathrm{N}+1$ electron wavefunction for any scattering state. This truncation approach effectively undermines what we think is a crucial point, namely, that the two electrons involved in the inelastic collisions should be treated on equal footing.

Fifth, sometimes the DWA is formulated with the use of different $U$ for the inftial state and for the final state. In dealing with discrete excitation, for example, it might appear natural to compute an initial distorted wave for a potential $U_{1}$ created by the initial (ground-state) target, and a final distorted wave for ancther potential $U_{f}$ created by the excited-state target. This idea (Implicit in the close-coupling approximation) might be appealing if one focuses on the behavior of the distorted waves at large distances. However, the interaction $V$ is strongest when the two electrons are close and, indeed, the riatrix element of Eq. 3.2 receives most of the contribıtions from the region niar or within the target radius. More importantly, the use of $U_{i}$ and $U_{f}$ leads to nonorthogonal sets of distorted waves and would introduce inconsistencies, as mentioned at the end of Sec. 3.

In practic ?, the difference between $U_{1}$ and $U_{f}$ is of the order $1 / 2$ compared to elther $U_{1}$ or $U_{f}$ itself, and should be small for large $z$. To Include corrections of the order $1 / z$, one should use correlated wavefunctions, perhaps along the lines of Sec. 5 , because it is decisive to describe accurately the motion of two electrons at short distances.

Indeed, recent DWA calculations on electron collisions with reutral atoms (Madison and Shelton 1973, Calhoun, Madison, and Shelton 1976, Thomas et al. 1974, Chutjlan and Thomas 1975, Baluja and laylor 1976) use a single $U$ for initial and final distorted waves, and are remarkably successful in reproducing experdmental results including angular distributions of inelastically 
scattered electrons and of ejected electrons. Moreover, an argument for the use of a single $U$ has been put forth by Rescigno et al. (1974).

The potential $U$ may include, in addition to the static-charge contribution, some polarization potential which accounts for the deformation of the target due to the incident electron. The polarization potential will be less important for larger $\mathrm{z}$, as well as for higher T. The properties of the polarization potential appropriate for low $\mathrm{T}$ and at large distances have been studied by Spruch and co-workers (see Berger, Snodgrass, and Spruch 1969, and references therein).

Finally, the most important criterion for the choice of $U$ is that it should give realistic phase shifts due to the short-range electron-core interactions. In this context, we must point out that even for highly stripped lons, phase shifts for low angular-momentum partial waves are gocd fractions of a radian and by no means negligible (Chao et al. 1976, and to be published). Therefore, correct evaluation of individual terms given by Eq. 3.2 must start with distorted waves with correct phase shifts.

However, it is widely belleved that the total fonization $\sigma_{1 \mathrm{cn}}{ }^{\prime}$ given as a sum of squares of many terms of the form Eq. 3.2, might be evaluated reasonably well by just 'ising pure Coulomb waves as distorted waves. This procedure is the Coulom:-Bom Approximation (CBA). The advantage of the CBA is that the pure Coulomb waves are analytically known and their properties can be readily studied. Thus, the CBA plays a fundamental role in electron-ion collisions comparable to the role of the plane-wave Born approxdmation in electron collisions with neutral atoms.

\subsection{Treatment in the Momentum Representation}

For high incident energies, the contributions to $\Omega_{\text {if }}$ from a wide band of $\ell_{0}$ values corresponding to large impact parameters should dominate. Furthermore, It is precisely under this circumstance that the DWA or the CBA becomes most reliable. Yet, the partial-wave summation then becomes impractical because of its slow convergence. Therefore, it is crucial to study the full inelastic-scattering amplitude for the DWA and the CBA from a point of view other than the partial-wave decomposition. 
Efforts in this direction (of obvious importance in our views) have been surprisingly scanty and fragmentary in the literature of atomic physics (Gailitis 1963). In this context, several papers on the Coulomb excitation of nuclei by charged particles (Guth and Mullin 1951, Mullin and Guth 1951, and Huby and Newns 1951) provide us with general ideas on the problem as sketched below.

In an altemative formulation of the DWA (or the $C B A$ ), we take the initial state $\vec{k}_{0} n_{i}$ and the final state $\vec{k}_{1} n_{f^{\prime}}$ specified by the incident-electron momentum $\hbar \vec{k}_{0}$ and by the scattered-electron momentum $\hbar \vec{k}_{1}$, respectively. Then, the differential cross section $d \sigma_{\text {If }}$ for the inelastic scattering into a solid-angle element $\mathrm{d} \omega$ around the direction defined by $\overrightarrow{\mathrm{k}}_{1}$ is expressed as ${ }^{*}$

$$
d c_{\text {If }}=\left(m^{2} / 4 \pi^{2} \hbar^{4}\right)\left(k_{1} / k_{0}\right)\left|\left(\vec{k}_{1} n_{f}|V| \vec{k}_{0} n_{1}\right)\right|^{2} d \omega .
$$

Evidently the essential dynamical factor is the matrix element $\left(\vec{k}_{1} n_{f}|v| \vec{k}_{0} n_{1}\right)$, taken between the initial and final states of the combined $\mathrm{N}+1$ electron system. Let us study the structure of this matrix element. For simplicity of discussion, we disregard the alectron-exchange term and concentrate on the direct-scattering term; the exchange tern could be added throughout.

We first express the Coulomb interaction $V$ between the incident electron at position $\vec{r}$ on the one hand and the target electrons at $\overrightarrow{r_{j}}$ on the other hand, as a Fourier integral

$$
V=\sum_{j=1}^{N} e^{2} i\left|\overrightarrow{r_{j}}-\vec{r}\right|=\left(2 \pi^{2}\right)^{-1} e^{2} \sum_{j=1}^{N} \int d \vec{q} q^{-2} \exp \left[-1 \vec{q} \cdot\left(\vec{r}-\overrightarrow{r_{j}}\right)\right] \text {. }
$$

Because the exponential function is factorized into $\exp (-i \vec{q} \cdot \vec{r})$ and $\exp \left(\overrightarrow{i q} \cdot \overrightarrow{r_{j}}\right)$, we may readily write

* The distorted-wave states $\vec{k}_{1}$ and $\vec{k}_{0}$ here should be normalized in a definite way to be consistent with Eq. 6.2. Expressed in the coordinate representation, their wavefunctions $x_{\vec{k}}{ }^{(t)}(\vec{r})$ are normallzed as

$$
\int \mathrm{d} \vec{r} \times \vec{k}^{(t) *}(\vec{r}) \times{\overrightarrow{k^{\prime}}}^{(t)}(\vec{r})=(2 \pi)^{3} \delta\left(\vec{k}-\overrightarrow{k^{\prime}}\right) \text {. }
$$

and are thus dimensionless. The plus sign refers to the outgoing-wave boundary condition, and the minus sign to the incoming-wave boundary condition. 


$$
\begin{aligned}
& \left(\vec{k}_{1} n_{f}|V| \vec{k}_{0} n_{i}\right)=\left(2 \pi^{2}\right)^{-1} e^{2} \int d \vec{q}^{-2}\left(\vec{k}_{1}|\exp (-i \vec{q} \cdot \vec{r})| \vec{k}_{0}\right) \\
& \times\left(n_{f}\left|\sum_{j=1}^{N} \exp \left(1 \vec{q} \cdot \vec{r}_{j}\right)\right| n_{i}\right) .
\end{aligned}
$$

The first matrix element $\left(\vec{k}_{1}|\exp (-1 \vec{q} \cdot \vec{r})| \vec{k}_{0}\right)$ is taken between the distorted waves (see footnote on p. 22). The second matrix element

$$
\left(n_{f}\left|\sum_{j=1}^{N} \exp \left(1 \vec{q} \cdot \overrightarrow{r_{j}}\right)\right| n_{1}\right) \equiv F_{1 f}(\vec{q})
$$

is taken between the target states. It is commonly called the (target) form factor, a quantity famlliar in the Bethe theory (Inokuti 1971).

The integrand at each value of $\vec{q}$ in $\mathrm{Eq} .6 .4$ represents the transfer of momentlim $\hbar \vec{q}$ from the incident electron to the target electrons. For further clarification of the meaning of Eq. 6.4, it is useful to consider the (planewave) Born approximation. Then, the first inatrix element reduces to

$$
\left(\vec{k}_{1}|\exp (-i \vec{q} \cdot \vec{r})| \vec{k}_{0}\right)=(2 \pi)^{3} \delta\left(-\vec{k}_{1}-\vec{q}+\vec{k}_{0}\right) \text {. }
$$

In other words, the contribution.s to the $\vec{q}$-integration are restricted to the unique value $\vec{q}=\vec{k}_{0}-\vec{k}_{1}$. The vector $\hbar \vec{K}=\hbar\left(\vec{k}_{0}-\vec{k}_{1}\right)$ is the momentum transfer for the overall process. Thus, we immediately obtain

$$
\left(\vec{k}_{1} n_{f}|V| \vec{k}_{0} n_{1}\right)=4 \pi 4^{2} K^{-2} F_{1 f}(\vec{K}) \text {. }
$$

Inserting Eq. 6.7 into Eq. 6.2 and expressing the solid-angle element $d \omega$ in terms of the magnitude $\hbar K$ of the momentum transfer as $d \omega=\pi d\left(K^{2}\right) / k_{0} k_{1}$, we can write

$$
\mathrm{d} \sigma_{\text {If }}=4 \pi \mathrm{k}_{0}^{-2} \omega_{i}^{-1}\left|\mathrm{~F}_{\mathrm{if}}(\mathrm{K})\right|^{2} \mathrm{~d}\left(\mathrm{Ka}_{0}\right)^{2} /\left(\mathrm{Ka}_{0}\right)^{4}
$$

Here we imply the customary averaging over the target orientation (alternatively over the magnetic quantum number of the target inftial state). The squared form factor after the averaging depends upon the scalar varlable $\mathrm{K}$ only, and is therefore written as $\left|\mathrm{F}_{\text {if }}(\mathrm{K})\right|^{2}$.

Integrating over all the possible values of $\left(\mathrm{Ka}_{0}\right)^{2}$ consistent with the energy conservation, we obtain 


$$
\left.\Omega_{1 \mathrm{f}}=\omega_{1}(\mathrm{~T} / \mathrm{R})\left(\sigma_{1 \mathrm{f}} / \pi \mathrm{a}_{0}^{2}\right)=4 \int\left|\mathrm{F}_{\mathrm{if}}(\mathrm{K})\right|^{2}(\mathrm{Ka})_{0}\right)^{-4} \mathrm{~d}\left(\mathrm{Ka} \mathrm{a}_{0}\right)^{2},
$$

an expression for $t^{2}$.e collision strength $\Omega_{1 f}$ (Eq. 3.1), especially appropriate for high incident energles $T=\left(k_{0} a_{0}\right)^{2} R$. Notice that in the Bethe theory the Incident electron makes a transition from a momentum eigenstate to another, that concomitantly the target makes a transition from its elgenstate to anothcr as a result of sudden transfer of momentum $\hbar \mathrm{K}$, and that the response of the target is described by its form factor, $F_{\text {if }}(K)$.

Returning to the DWA (or the CBA), we express the distorted waves, $x^{\prime} s$, as

$$
x{\overrightarrow{k_{f}}}^{(-)}(\vec{r})=\int \overrightarrow{d p^{\prime}} \phi_{k_{f}}^{(-)}\left(\overrightarrow{p^{\prime}}\right) \exp \left(\overrightarrow{i p^{\prime}} \cdot \vec{r}\right)
$$

and

$$
x \vec{k}_{1}^{(t)}(\vec{r})=\int d \vec{p} \phi \vec{k}_{1}^{(t)}(\vec{p}) \exp (\overrightarrow{i p} \cdot \vec{r}) .
$$

using the momentum wavefunctions $\phi^{\prime} s$. By use of Eqs. 6.10 and 6.11 , we can write the first matrix element of Eq. 6.4 as

$$
\begin{gathered}
\left(\vec{k}_{1}|\exp (-1 \vec{q} \cdot \vec{r})| \overrightarrow{k_{0}}\right)=\int d \vec{r} \times \vec{k}_{1}^{(-)^{*}}(\vec{r}) \exp (-1 \vec{q} \cdot \vec{r}) \times \vec{k}_{0}{ }^{(+)}(\vec{r}) \\
=(2 \pi)^{3} \int d \overrightarrow{p^{\prime}} \int d \vec{p} \phi_{\vec{k}_{1}}(-)^{*}\left(\overrightarrow{p^{\prime}}\right) \phi_{\vec{k}_{0}}(\vec{p}) \delta\left(-\overrightarrow{p^{\prime}}-\vec{q}+\vec{p}\right) .
\end{gathered}
$$

Substitution of Eqs. 6.5 and 6.12 into Eq. 6.4 leads to

$$
\begin{aligned}
&\left(\vec{k}_{1} n_{f}|V| \vec{k}_{0} n_{1}\right)=4 \pi e^{2} K^{-2} \int d \overrightarrow{p^{\prime}} \int d \vec{p} w\left(\overrightarrow{p^{\prime}}, \overrightarrow{p_{i}} \vec{k}_{1}, k_{0}\right) \\
& \times F_{1 f}\left(\vec{p}-\overrightarrow{p^{\prime}}\right) .
\end{aligned}
$$

where we have defined

$$
w\left(\overrightarrow{p^{\prime}}, \vec{p} ; \vec{k}_{1}, \vec{k}_{0}\right)=\left|\vec{k}_{0}-\vec{k}_{1}\right|^{2}\left|\vec{p}-\vec{p}^{\prime}\right|^{2} \phi_{\vec{k}_{1}}^{(-)^{*}}\left(\overrightarrow{p^{\prime}}\right) \phi_{\vec{k}_{0}}^{(+)}(\vec{p}) .
$$

Compare this result with the (plane-wave) Bom-approximation result, 1.e. ,

Eq. 6.7. The use of the distorted waves amounts to redistributing the momentum transfer according to the weight $w\left(\overrightarrow{p^{\prime}}, \overrightarrow{p_{i}} \vec{k}_{f}, \vec{k}_{1}\right)$, but the response of the target to individual values of $\vec{p}-\overrightarrow{p^{\prime}}$ remains described by the same form factor. Notice 
at the same time that the superposition of target responses described by Eq. 6.14 applies to the scattering amplitude, as opposed to the cross section.

We now carry out the same procedure as in the Bethe theory; the final result is

$$
\Omega_{\text {if }}=4 \int \Phi_{\text {if }}\left(\vec{k}_{0}, \vec{k}_{1}\right)\left(\mathrm{Ka}_{0}\right)^{-4} \mathrm{~d}\left(\mathrm{Ka}_{0}\right)^{2},
$$

to be compared with Eq. 6.9, where we have defined

$$
\Phi_{i f}\left(\vec{k}_{0}, \vec{k}_{1}\right)=\left|\int d \overrightarrow{p^{\prime}} \int d \vec{p} w\left(\overrightarrow{p^{\prime}}, \vec{p} ; \vec{k}_{1}, \vec{k}_{0}\right) \vec{s}_{\text {if }}\left(\vec{p}-\vec{p}^{\prime}\right)\right|_{A v}^{2} .
$$

Again we imply the customary averaging over the target orientation. The suffix "Av" on the right-hand side of Eq. 6.16 is meant to remind us of this procedure. Instead of $\left|F_{\text {if }}(K)\right|^{2}$ in Eq. 6.9, we now have in Eq. 6.15 the quantity $\Phi_{\text {if }}\left(\overrightarrow{k_{1}}, \overrightarrow{k_{f}}\right)$. According to Eq. 6.16 , this quantity is a sum of $F_{\text {if }}^{\star}\left(\vec{p}-\vec{p}^{\prime}\right) F_{\text {if }}\left(\vec{q}-\overrightarrow{q^{\prime}}\right)$ weighted by the factor $w=w^{*}\left(\overrightarrow{p^{\prime}}, \vec{p} ; \vec{k}_{1}, \vec{k}_{0}\right) w\left(\vec{q}, \vec{q} ; \vec{k}_{1}, \vec{k}_{0}\right)$, which is essentially a product of four momentum wavefunctions for the distorted waves. Recall that in the (plane-wave) Born approximation, $W=\delta\left(\overrightarrow{p^{\prime}}-\vec{k}_{1}\right) \delta\left(\overrightarrow{q^{\prime}}-\vec{k}_{1}\right) \delta\left(\vec{p}-\vec{k}_{0}\right) \delta\left(\vec{q}-\vec{k}_{0}\right)$. The DWA (or the CBA) amounts to taking into account a "blurring" of the momentum transfer due to the distortion potential $U$, as well as interference between the contributions of altemative transfers $\vec{p}-\overrightarrow{p^{\prime}}$ and $\vec{q}-\overrightarrow{q^{\prime}}$.

Consequently, the weight function $w$ must be the object of detailed investigation and quantitative evaluation. However, we may now point out a few of its elementary properties and their implications. By far the most $1 \mathrm{~m}$ portant property is that $w$ maintains delta-function singularities at $\overrightarrow{p^{\prime}}=\vec{k}_{1}$, $\vec{p}=\vec{k}_{0}$ in the DWA in general. In the CBA in particular, its analytical form is not only completely known, but is also rather simple (ruth and Mullin 1951 and Mullin and Guth 1951). The other parts of $w$ are obviously less important with regard to the integrated cross section $\sigma_{\text {if }}$ (or to the collision strength $\Omega_{1 f}$ ), and may be studied in some detail by a perturbative treatment of the dis torted potential $\mathrm{U}$ at least for high incident electron energies.

The Glauber approximation and other similar approximations (Gerjuoy and Thomas 1974) may be also considered as variants of the DWA. In this class of treatment, Initial- and final-state distorted waves are generated from 
particular prescriptions, which amount to a special choice of $U$ (which may be non-local or $\mathrm{T}$ dependent, or both). The circumstance sometimes makes it difficult to identify what kind of physical mechanisms a:a included or excluded from any particular formulation. An analysis of the Glaubsr approximation (and of its variants) along the lines of the present treatment might shed light on its physical meaning.

\subsection{Additional Remarks}

We supplement the foregoing discussion by making several technical points.

First of all, le: us consider the CBA, specifically when the Coulomb field (Eq. 6.1) is weak. More precisely, when $k_{0} a_{0} \gg z, k_{1} a_{0} \gg z$, and $z$ is not very large, the value of the form factor $F_{i f}(\vec{p}-\vec{p})$ contributing to the integral of Eq. 6.13 is effectively put as $F_{1 f}(K)$, because the weight factor $w\left(\overrightarrow{p^{\prime}}, \vec{p}\right.$; $\vec{k}_{1}, \vec{k}_{0}$ ) is much more strongly dependent on $\overrightarrow{p^{\prime}}$ and $\vec{p}$ (as exemplified by its delta-function singularities at $\overrightarrow{p^{\prime}}=\vec{k}_{1}$ and $\vec{p}=\vec{k}_{0}$. Then, we an the form factor cut of the integral and write

$$
\begin{gathered}
\left(\vec{k}_{1} \Omega_{f}|v| \vec{k}_{0} n_{1}\right) \cong 4 \pi e^{2} K^{-2} F_{i f}(K) \int d \overrightarrow{p^{\prime}} \int d \vec{p} w\left(\overrightarrow{p^{i}}, \vec{p} ; \vec{k}_{1}, \vec{k}_{0}\right) \\
\cong 4 \pi e^{2} K^{-2} F_{\text {if }}{ }^{(K)} \times{\overrightarrow{k_{1}}}^{(-) *}(0) \times \vec{k}_{0}{ }^{(+)}(0) .
\end{gathered}
$$

where $x(0)$ 's represent the value of the distorted waves (In the coordinate representation) at $r=0$, as discussed by Guth and Mullin (1951). Under this special circumstance, the differential cross section given by Eq. 6.2 reduces to the plane-wave Boin approximation result (Eq. 6.8) multiplled by

$$
\begin{aligned}
\eta & =\left|x \vec{k}_{1}^{(-) *}(0) \times \vec{k}_{0}^{(+)}(0)\right|^{2} \\
& =\frac{4 \pi^{2} z^{2}}{\left(k_{1} a_{0}\right)\left(k_{0} a_{0}\right)\left[1-\exp \left(-2 \pi z / k_{1} a_{0}\right)\right]\left[1-\exp \left(-2 \pi z / k_{0} a_{0}\right]\right]}
\end{aligned}
$$

1.e., the Elwert factor well known in the bremsstrahlung theory. Th1s factor certainly is close to unity under our conditions, but its approach to unity for greater and greater $k_{0}$ (and $k_{1}$ ) is quite gradual. This result suggests that the 
combination Tu $/ \eta$ (Instuad of To, might be a quanilty sultable for consideration on the asymptotic high-energy behavior of $\sigma_{f}$

Second, cioss connection o! the DViA to the bremsstrahlung theory can be made apparcent under differont conditions, too. Lsi us considar the contributions (to the integral of $E_{7}$. 6.4) from small values of $q$, which must domlrate at high $T$ because of the $q^{-2}$ factor in Eq. 6.4. Expressed in nore conventional terms, these contributions item from collisions with large-impact parameters (as discussed in Sec. 4). Then, we may expand bott, exponentials in Eq. 6.4 into the power series and retain the first non-vanishing terms only: the result is

$$
\left(\vec{k}_{1} n_{f}|v| \vec{k}_{0} n_{l}\right)=\left(2 \pi^{2}\right)^{-1} e^{2} \int d \vec{q} \frac{1}{3}\left(\vec{k}_{1}|\vec{r}| \vec{k}_{0}\right) \cdot\left(n_{f}\left|\sum_{j=1}^{N} \vec{r}_{j}\right| n_{1}\right) \text {. }
$$

The first factor on the right-hand side is nothing but the dipole matrix element for a bremsstrahlung prozess in which the incident electron makes the transition from state $\vec{k}_{0}$ to state $\vec{k}_{1}$ in the field $U$. An analytical expression for this matrix element $\left(\vec{k}_{1}|\vec{r}| \frac{1}{k_{0}}\right)$ for the CBA is well known since the pioneering work of the Sommerfeld school (Sommerfeld 1947), as S. Geltman kindly pointed out to M.I. in 1970. The second factor is even more famillar to us; it is the dIpole matrix element $x_{\text {if }}$ for the target transition $n_{1} \rightarrow n_{f}$, which appears in Eq. 2.3.

An analysis of the properties of the bremsstrahlung matrix element $\left(\vec{k}_{1}|\vec{r}| \vec{k}_{0}\right)$ (by Inokutd and $\mathrm{KIm}$, unpublished) has so far led to a conclusion on the asymptotic behavior of the collision strength $\Omega_{1 f}$ (Eq. 2.3), namely, that the coefficient of the leading loganthmic term at high $\mathrm{T}$ remains the same (1.e., $4\left|x_{1 f} / a_{0}\right|^{2}$ ) in the CBA as in the (plane-wave) Bom approximation. At the same time, the constant $c_{1 f}$ must be modifled when one goes from the Bom approximation to the CBA or to any versions of the DWA.

Finally, the recent and extensive work of Pratt and his co-workers (Pratt and Tseng 1975 and Lee et al. 1976) on the bremsstrahluny process is important for full understanding of the matrix element $\left(\vec{k}_{1}|\vec{r}| \vec{k}_{0}\right)$. The principal merit of their work is seen in the use of distorted waves las opposed to pure 
Coulomb waves) computed for a realistic distortion potential $U$, and in the development of analytical and numerical techniques especially suitable at high incident-electron energies.

\section{Inner-Shell Ionization}

Inner-shell processes are secondary to the main theme of the present report, but a few remarks concerning them serve to supplement our discussion.

The first remark is that a collision with an inner-shell electron depends but little on the presence of shells extemal to it, unless one of the electrons escapes with low energy. The influence of external shells on the electron escape has been treated by Fano. Theodosiou, and Dehmer (1976) for neutral atoms. Analugous studies for ions are in progress (Chao et al. 1975). For the photoeffect in Ions, see Reilman (1974).

Accordingly, one may compare the lonization of an inner shell to that of the same shell in an Ion from which all external shells have been stripped. Inner-shell ionization processes should then be generally amenable to calculation by a lowest-order perturbation treatment, that is, according to Sec. 6 , by Coulomb-Bom, or rather, by the distorted-wave approxdmation. In any event, the wavefunction of the incident electron should be strongly affected by the ionic fleld in these processes, especially for incident energies, $T$, of the same order as the relevant Ionization threshold I; then, the impact parameter cannot be much larger than the lonic radius.

There is a sizable amount of experimental data on inner-shell ionization by electron collisions (Powell 1976) for two reasons; first, $x$ rays resulting from inner-shell Ionization are often easy to measure, and second, inner-shell Ionization cross sections are needed for many applications such as $x$-ray fluorescence, chemical analysis and Auger-electron spectroscopy. On the grounds described in the previous paragraph, the relative richness of experimental data on inner-shell Ionlzation should be taken as an advantage for leaming about electron collisions with highly stripped ions, a subject that is difficult to study experimentally. 
A remarkable fact, however, is that fair agreement with experiment does emerge from calculations of inner-shell ionization performed by the plane-wave Bom approximation, without any allowance for distortion of the incident electron's wavelunction, for values of $\mathrm{T}$ as low as $\sim 4 \mathrm{I}$. This circumstance has been noticed for decades but does not appear to have been explained or even discussed extensivisly. It is now documented by extensive avidence in Powell's review (1976).

Powell's observations as quoted above require some qualifications. More precisely speaking, $\Omega_{1 f}$ for $K$ - and L-shell iontzation of many atoms shows the $\mathrm{T}$ lependence to be analytically the same as given by Eq. 2.3 for $\mathrm{T}>4 \mathrm{I}-$ a condition necessary (though not sufficlent) for the applicability of the Bom approximation. But the coefficient in front of the logarithms determined from experimental $\Omega_{\text {if }}$ tends to be greater than the value expected from independent measurements on photoabsorption or from theory. This makes Powell suspect that the true asymptotic behavior (Eq. 2.3) may not necessarlly have been attained in the range of $\mathrm{T}$ of many measurements (1.e., $\mathrm{T}<60 \mathrm{I}$ ).

Within the framework of Eq. 3.1, it appears certain that the atomic field would seriously influence the values of individual terms of the summation, for specific values of $\ell_{0}$, by distorting the incident electron's wavefunction, especially in perturbative calculations for $T<4 \mathrm{I}$. Insofar as the results obtained with undistorted wavefunctions are adequate, we argue that extensive compensation of errors is achieved somehow by summing terms of Eq. 3.1. Equation 3.1, which represents the transition probability as the sum of contributions from transitions between states labeled by angular momentum quantum numbers, might conceivably be transformed into a sum of contributions with different labels less sensttive to distortion of the wavefunctions.

This problem may be related to the final remark of Sec. 4 on the contrast between integrated cross sections and differential cross sections. [Madison (1976) emphasizes the merit of studying differential cross sections, 1.e., the en 3rgy and angular distributions of ejected electrons, as well as of scattered electrons specifically for inner-shell ionlzation.] No specific suggestion to this effect seems to have been presented. The problem thus presented remains 
unsolved and puzzling.

The search for a solution to this problem might perhaps be guided by the successful treatment of inelastic collisions by low-velocity heavy particles. In this case, the ordinary Bom treatment is replaced by a first-order perturbation troatment in which the incident particle is assumed to follow a classical rectilinear trajectory, or also a trajectory deflected by the atomic field. In elther case, the result coincides, in essence, with that of the (plane-wave) Bom approximation. The reasons for the invariance of the results with respect to major changes of the model do not appear to have been investigated in detail. They might relate to the well-known coincidence of the classical and quantum mechanical (Bom or exact) formulas for Ritherford scattering.

\section{Penpheral Remarks}

Most of the ionizing collisions at low-to-moderate energy generally result in the ejection of valence shell electrons, malrily because valence orbitals present the largest target to incident electrons. A notable exception to this rule occurs for Ionization of the heavier alkali- and alkaline-earth-like isoelectronic sequences above the threshold for excitation of the next inner subshell with an $\mathrm{np}^{6}$ structure (Peart and Dolder 1975). One contributing factor is that this inner shell is itself rather large, has a low excltation threshold, and is occupied by six electrons vs. one or two in the valence shell. A second factor stems from the circumstarce that, whereas the excitation of a valence electron to a $p$ state of the same shell has an unusually large cross section for low energy collisions (Leep and Gallagher 1976; Chen, Leep, and Gallagher 1976) (or photoabsorption), the cross section decreases rapidly for higher excitations and becomes very low for ionizations. The concentration of the transition probabllity into the lower excitation channels, at expense of Ionization, extends to all target electrons as one proceeds from neutrals to highly stripped lons. This important change of atomic properties with increasing degree of ionization results from the approach to the Coulomb-fleld properties, and has been demonstrated by pilot calculations. It was foreshadowed by a demonstration that most of the ionization of highly stripped lons occurs by the two-step process 
of Inner-shell excitation followed by Augar effocts (Hahn and Watson 1973 and Hahn 1976).

Our attention has centered in this report on the total cross sections for Ionization. From this point of view, the spectral and angular distributions of electrons ejected in ionizing collisions are only of subsidiary interest. Yet their study may provide important clues, or at least confirmations, of the underpinning of the approximate calculations of total cross sections. High-energy collisions are usually classified into "glancing" ones - with low momentum transfer 4 , and low or moderate energy transfer and dipole-like angular distrbution of ejected electrons - and into "binary" or "quasi-free" collistons, in which the ejected electron hardly interacts with its initial atom and recolls with appreciable momentum $q$ and with kinetic energy $E \sim q^{2} / 2 m$. KIm (1975) has recently shown how to identify asfects of this classification even in extensive data on low-energy collistons. The identification is particularly striking in the IImited but very detalled data obtained by Ehrhardt and co-workers (Ehrhardt et al. 1972, 1974, and Jung et al. 1975) by coincidence detection of the scattered and ejected electrons. Theoretical account of their distributions has, however, been only fragmentary. Glancing collisions usually predominate over quasi-free collisions, at least at high energles, but exceptions do occur.

\section{Raforences}

Boluja, K. L. and H. S. Taylor, 1975, J. Phys. B $\underline{9}, 229$.

Berger, R. O., H. B. Snodgrass, and L. Spruch, 1969, Phys. R.av. 185, 113.

Burke, P. G. and M. J. Seaton, 1971, Methods in Computatlonal Physics, Vol. 10, edited by B. Adler, S. Fembach, M. Rotenberg (Academic Press, New York), p. 1.

Burke, P. G. and W. D. Roob, 1975. Aúvances In Atomic and Molecular Physlcs, Vol. 11, adited by D. R. Bates and B. Bederson (Academic Press, New York), p. 143.

Burke, V. M. and M. J. Ssaton, 1901, Proc. Phys. Soc. 77, 149.

Calhoun, ... V., D. H. Madison, and W. N. Shelton, 1976, Ex:itation of the $n=2$ state of atomic hydrogen by uleriron impact in the distorted-wave approximation, a preprint.

Chang, T. N. and R. T. Poe, 1975, Phys. Rav. A $\underline{12}, 1432$. 
Chao, K. D., J. L. Dehmer, U. Fano, M. Inokitt, S. T. Minson, A. Msezalie, R. F. Rasilman, and C. E. Theodosiou, 1075, Proceedings of the IVth Intemational Conference on Beam-Foll Spectroscopy and Heavy-Ion Alomic Physics, Gatlinburg, Tennesses, Saptember 1975 to be published by Plenusu Publishing Corp.).

Chen, S. T., D. Leep, and A. Gallaghar, 1975, Phis. R.:v. A 13., 947.

Chutjian, A. and L. D. Thomas, 1975, Phys. Rev. A $11,1583$.

de Hear, F. J. and R. H. J. Jansen, 1975, Total Cross Sactlons for Electron Scuttering by $\mathrm{Ne}, \mathrm{Ar}, K_{r}$, and $X g$, FOM-Report No, 37.174.

Ehrhardt, H., K. H. Hesselbacher, K. Jung, and K. Willmann, 1972, Case Studles in Atomic Collision Physics II, edited by E. W. McDanlel and M. R. C. McDowell (North-Holland Publishing Co., Amsterdam), p. 159. Enrhardt, H., K H. Hesselbacher, K. Jung, E. Schubert, and K. Willmann, 1974, J. Pinys. B 7, 69.

Fano, U., 1954, Phys. Rav. 95, 1198.

Fano, U., 1975, Radiat. Res. E⿺辶. 217.

Fano, U., C. E. Theodosiou, and J. L. Dehmer, 1976, Rev. Mod. Pnys. 48, 49.

Gallitis, M. K., 1966, Atomic Collisions, edited by V. Ya. Valdre, R. Ya. Dumburg, and R. K. Peterkop, translated by M. V. Kurepa, (Butterworths, London), pp. 81 and 87.

Gerjuoy, E. and B. K. Thomas, 1974, Rept. Prog. Pnys. 37, 1345.

Guth, E. and C. J. Millin, 1951, Phys. Rev. 83, 667.

Hahn, Y. and K. M. Watson, 1973, Phys. Rev. A $1,491$.

Hahn, Y., 1976, Phys. Rev. A $13,1326$.

Huby, R. and H. C. Newns, 1951, Proc. Pnys. Soc. (London) A64, 619. Inokuti, M., 1971, Rev. N:od. Phys. 43, 297.

Jung, K., E. Schubert, D. A. Paul, and E. Ehrhardt. 1975, J. Phys. B $\underline{8}, 1330$. Kim, Y. $-K ., 1975$, Radiat. Res. 61, 21; 64, 95.

Kinı, Y. -K., M. Naon, and M. Comille, 1973, in Argonne National Laboratory Radiological and Environmental Research Division Annual Report, July 1972-June 1973, Report No. ANL-8060-I, p. 14.

Korif, D. F., S. Chung, and C. C. Lin, 1973, Phys. Rev. A I, 545.

Layzer, D., 1959, Ann. Phys. (New York) $8,271$.

Loe, C. M. , 1974, Phys. Rev. A 1․․, 584.

Lee, C. M., L. Kissel, R. H. Pratt, and H. K. Tseng, 1976, Phys. Rev. A 13 , i714.

Leep, D. and A. Gallagher, 1976, Phys. Rev. A $13,148$.

M:- Iison, D. H. , 1976, Invited lecture at the Second Intemational Conference on Inner-Shell Ionization Phenomena, Frelburg, Germany, 29 March2 April 1976, to be published.

Madison, D. H. and W. N. Shelton, 1973, Phys. Rev. A Z, 499.

Molselwitsch, B. L. and S. J. Smith, 1968, Rev. Mod. Phys. 40, 238.

Mott, N. F. and H. S. W. Massey, 1965, The Theon of Atomic Collisions,

Third Edition (Oxford University Press, London).

Mullin, C. J. and E. Guth, 1951, Phys. Rev. 82, 141.

Peart, B. and K. Dolder, 1975, J. Phys. B $\underline{8}, 56$. 
Powell, C. J., 1976, Rev. Mod. Phys. 48, 3.3.

Pratt, R. H. and H. K. Tseng, 1975, Phys. Rev. A 11, 1797.

Rellman, R. F., 1974, M.S. Thesis, Georgla State University.

Rescigno, T. N., C. W. McCurdy, Jr., and V. McKoy, 1974, J. Phys. B ?, 2396.

Rudige, M. R. H., 1968, Rev, Mod. Phys. 40, 564.

Seaton, M. J., 1953, inil. Trans. Roy. Soc. A245, 469.

Seaton, M. J., 1955, Proc. Phys. Soc. A 68, 457.

Seaton, M. J., 1961, Proc. Phys. Soc. 77, 174.

Seaton, M. H. , 1975, Advances in Atomic and Molecular Physics Vol. 11, edited by D. R. Bates and B. Bederson (Academic Press, New York), p. 83.

Sommerfeld, A., 15.7, Wellenmechank, (F. Ungar Pub. Co., New York). A reprint of the second revised anc enlarged edition, 1939, of Vol. 2 of the author's Atombau und Spektrallinien, originally published in 1929 as Wellenmechänlscher Ergänzungsband, Chapter 7.

Thomas, L. D. , G. Csanak, H. S. Taylor, and B. S. Yarlagadda, 1974, J. Phys. B ?, 1719.

Vainshtein, L. A. and L. P. Presnyakov, 1968, Zh. Eksp. Teor. Fiz, 55, 297 (Sov: Phys. -JETP 28, 156, 1969).

Mi, T. -Y. and T. Ohmura, 1962, Quantum Theory of Scattering (Prentice-Hall, Inc., Englewood Cliffs, N.J.). 
APPENDIX : NOTE ON EXTENDING PUANTUM DEFECT THEORY TO MULTIPLE EXCITATIONS*

\section{Introduction}

The Quantum Defect Theory (QDT) has proven very effective in predictIng and correlating a large variety of spectral and collision properties of atoms and molecules. Its formalism, developed mainly by Seaton, ${ }^{1}$ exploits the following circumstances. The wavefunction of an excited electron in the Coulomb field that surrounds an ionic core is a solution of the Schroedinger equation for a $\mathrm{H}$ atom; it is thereby known throughout this region in analytic form, to within parameters which may be identifled by boundary conditions. The excited electron need not remain in this region, but it may also dip into the ionlc core whele it experiences complex interactions with other electrons; the net effect of these interactions upon the wavefunction in the outer Coulomb field may be represented by imposing on this wavefunction approprate boundary conditions on a surface that envelops the core. These conditions can be determined either by $a b$ initio solution of the Schroedinger equation within the cure or by semiempirical analysis of experimental data. This point of view has been fllustrated recent$1 y^{2}$ with reference to numerous applications.

It is conceptually important that the complicated problem of the electron's interaction within the core is reduced by the QDT to the solution of the Schroedinger equation within a finite volume. The elgenstates of this problem form a discrete set, yet serve equally to represent properties of discrete and continuum states of the system "core + outer fleld." Exploitation of this feature underlies recent promising progress by alternative, but equivalent, $R$-matrix techniques. 3,4

Both the QDT and the R-matrix concepts have, however, been restricted to dealing with a single particle emerging from a core. Double, or multiple,

* This note by U. Fano has been circulated informally for information and comment with no plan for early publication. January 1976)

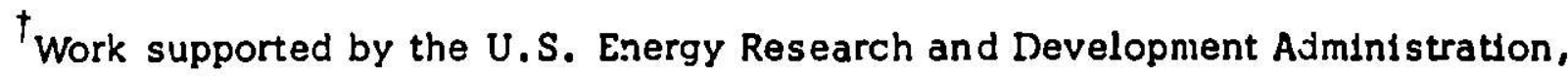
Contract No. COO-1674-116. 
excitations result not infrequently from photoabsorption or fâst collisions. More importantly, they result normally in the course of inelastic collisions of slow electrons leading to discrete excitation or to ionization, 1.e., whenever an initial atom or molecule is augmented by an incident slow electron which then re-emerges together with an electron ejected from the initial target volume. Lack of a proper treatment of the latter process constitutes a major shortcoming of current atomic theory.

Th1s paper surveys the problems to be solved in order to extend the QDT and R-matrix procedures to processes where two (or more) electrons emerge from an ionic core. It also provides a solution to one of these problems.

We consider three separate, though interrelated, problems:

a) Construction of wavefunctions for two (or more) electrons in the Coulomb field of an ionic core. If a wavefunction is identified by its boundary properties on a surface surrounding the core, its behavior at infinite distance from the core determines the scattering matrix or equivalent observable properties of the atomic system. Conversely, if a wavefunction is identified by its behavior at infinity, on the basis of experimental data, its boundary properties on a surface near the core depend on energy weakly and smoothly, and therefore afford a compact representation of experimental data. ${ }^{2}$ The relationship between boundery properties near the core and at infinity is the backbone of the QDT. For a single electron, this relationship is derived from our knowledge of analytic Coulomb field wavefunctions. ${ }^{1}$ Obtaining the analogous relationship for two electrons constitutes a serlous hurdle.

This problem has been approached following the early experimental and computational studies of doubly excited helium states. ${ }^{5}$ Since a He atom consists of two electrons in the field of a point nucleus, rather than of an lonic core of finite size, its wavefunctions should serve in a QDT treatment of double excitation of other atoms in the same capacity as the wavefunctions of a $\mathrm{H}$ atom, with a point nucleus, serve in a QDT treatment of single excitation of other atoms. Considerable progress has been achieved in surveying the properties of doubly exclted He wavefunctions and in studying them numerlcal1y. ${ }^{6}$ This progress is not yet adequate to provide a manifold of two-electron 
wavefunctions with the extent and accuracy required for quantitative QDT applications, but it permits the preliminary analysis at which we aim here.

b) Formulation of boundary conditions on a surface surrounding the core. Solutions of wave equations, obtained separately in two different regions of space, are joined at the boundary of such regions by matching the normal logarithmic derivatives and the amplitude variations of the two solutions over the whole boundary. In the QDT of single-electron excitations, the boundary is a spherical surface, the amplitude varlations over this surface are expanded into sptırical harmonics and the noimal (1.e., radial) logarithmic derivative may be expressed in terms of a quantum defect. The extension of this formulation to two-electron excitations forms the main subject of the present paper.

c) Solution of the wave equation within the ionic core. The conceptual appruach to this problem outlined in Ref. 4(a) is equally applicable, in princlple, when two or more electrons ran reach the boundary. Implementation of the approach depends, however, on the formulation of boundary conditions and will only be indicated here.

Section II will summarize relevant current knowledge of two-electron Coulomb functions. Section III will deal with the boundary conditions, 1.e., with problem b), and Section IV will comment on the special but important case where the system "core +2 electrons" has a net negative charge.

\section{Two-Electron Coulomb Functions}

The recent study of these functions has been motivated and guided by the experimental discovery of novel classification and selection rule schemes for doubly excited helium states $\left(\mathrm{He}^{* \star}\right){ }^{5}$ These schemes were interpreted initially, but only rather superficlally, as effects of configuration interaction, 1.e., in terms of superposition of independent-electron wavefunctions. Attention was then directed to studying wavefunctions in the 6 -dimensional $\left(\vec{r}_{1}, \vec{r}_{2}\right)$ configuration space, seeking coordinates that might prove quasi-separable. Quantum numhers conjugate to such coordinates would then represent quasiInvariants and serve to classify the Rydberg serles of levels and the adjoining continua which constitute the observed channels of double excitation. 
The hyperspherical ("Fock") coordinates appear to fill this role. ${ }^{6}$ They result, in the main, by replacing the radial coordinates of an electron pair $\left(r_{1}, r_{2}\right)$ by a hyperradius $R=\left(r_{1}^{2}+r_{2}^{2}\right)^{\frac{1}{2}}$ and a mock-angle $\alpha=\arctan \left(r_{2} / r_{1}\right)$, shown in Fig. 1. Whereas the dependence of the wavefunction on $r_{1}$ or $r_{2}$ characterizes the radial motion of one or the other electron, its dependence on $\mathrm{R}$ describes, in essence, a Joint radial motion of the electron pair, while the dependence on $\alpha$ describes alternating (i.e., out of phase) variations of $r_{1}$ and $r_{2}$. That is, the coordinates $\left(r_{1}, r_{2}\right)$ are appropriate for describing uncorrelated radial motions of the electrons, while $(R, \alpha)$ serve when correlations are strong and the joint and alternating motions are more nearly independent of one another. The remaining four coordinates, $\hat{r}_{1} \equiv\left(\theta_{1} \phi_{1}\right)$ and $\hat{r}_{2} \equiv\left(\theta_{2} \phi_{2}\right)$, are not essential for our immediate purposes, but our diagrams in the $\left(r_{1}, r_{2}\right) \equiv(R, \alpha)$ plane should be understood to represent sections of the full space for an unspecified set of values of the other coordinates. In these coordinates and in atomic units, the Schroedinger equation takes the form

$$
\begin{gathered}
\left\{\frac{\partial^{2}}{\partial R^{2}}+\left[\frac{1}{R^{2}}\left(\frac{\partial^{2}}{\partial \alpha^{2}}-\frac{\vec{l}_{1}^{2}}{\cos ^{2} \alpha}-\frac{\vec{l}_{2}^{2}}{\sin ^{2} \alpha}-\frac{1}{4}\right)+\frac{C\left(\alpha, \theta_{12}\right)}{R}\right]\right. \\
+2 E\}\left(R^{\frac{5}{2}} \sin \alpha \cos \alpha \Psi\right)=0,
\end{gathered}
$$

where $\vec{l}_{1}$ and $\vec{l}_{2}$ indicate the orbital momenta of the two electrons,

$$
\mathrm{C}\left(\alpha, \theta_{12}\right)=2 \mathrm{Z} / \cos \alpha+2 \mathrm{Z} / \sin \alpha-2 /\left[1-\sin 2 \alpha \cos \theta_{12}\right]^{\frac{1}{2}}
$$

represents the whole potential energy multiplied by $-R$, with $\cos \theta_{12}=\hat{r}_{1} \cdot \hat{r}_{\text {?' }}$ and $E$ is the energy elgenvalue; the factor $R^{\frac{5}{2}} \sin \alpha \cos \alpha$ serves to eliminate first derivatives.

One may consider solutions of Eq. 1 at three successive levels of approximation, with the goal of representing each exact solution as consisting mainly of one approximate solution with a minor admisture of others:

a) The zero-th approximation consists of Lin's wavefunctions ${ }^{6}$

$$
R^{\frac{5}{2}} \sin \alpha \cos \alpha \Psi \sim \Phi_{\ell_{1} \ell_{2} m}\left(R ; \alpha, \hat{r}_{1}, \hat{r}_{2}\right) F_{E}^{\left(l_{1} l_{2} m\right)}
$$


with

$$
\begin{gathered}
\Phi_{\ell_{1} \ell_{2} m}\left(\mathrm{R} ; \alpha, \hat{\mathrm{r}}_{1}, \hat{\mathrm{r}}_{2}\right)=\mathrm{g}^{\ell_{1} \ell_{2}} \mathrm{~m}(\mathrm{R} ; \alpha) \mathrm{Y}_{\ell_{1} \ell_{2} L M}\left(\hat{\mathrm{r}}_{1}, \hat{\mathrm{r}}_{2}\right) \\
+(-1)^{S} \mathrm{~g}_{\mathrm{m}}^{\ell_{1} \ell_{2}}\left(\mathrm{R} ; \frac{1}{2} \pi-\alpha\right) \mathrm{Y}_{\ell_{1} \ell_{2} \mathrm{LM}}\left(\hat{\mathrm{r}}_{2}, \hat{\mathrm{r}}_{1}\right)
\end{gathered}
$$

in which the coordinates are fully separated except for antisymmetrization and orbital coupling. Here $\mathrm{Y}_{\ell_{1} \ell_{2} L M}$ indicates a product of spherical functions of $\hat{r}_{1}$ and $\hat{r}_{2}$, with orbital momenta $\ell_{1}$ and $\ell_{2}$ coupled to $(\mathrm{LM}), g^{\ell_{1} l_{2}}$ (R; $\left.\alpha\right)$ indicates a solution of the partial wave equation in $\alpha$, with m nodes, evaluated for fixed $R$ and fixed $\left(\ell_{1} \ell_{2} L M\right)$, and $S$ is the spin quantum number which characterlzes the permutation symmetry of $\mathrm{Eq}$. 3. Also, $\mathrm{F}_{\mathrm{E}}$ indicates a solution of the radial equation which is obtained from Eq. 1 by entering in $\mathrm{It} \mathrm{Eq} .3$, multiplyIng by $\Phi_{\ell_{1} \ell_{2} m}\left(R ; \hat{r}_{1} \hat{r}_{2} \alpha\right)$ and integrating over $\hat{r}_{1}, \hat{r}_{2}$ and $\alpha$. Note that I.In's 6 -variable wavefunction has exactly six quantum numbers $\left(l_{1} \ell_{2} \mathrm{~L} \mathrm{MmE}\right)$, as one expects for full separation of its variables.

b) The adiabatic approximation of $\mathrm{Macek}^{7,5}$ which replaces the functions

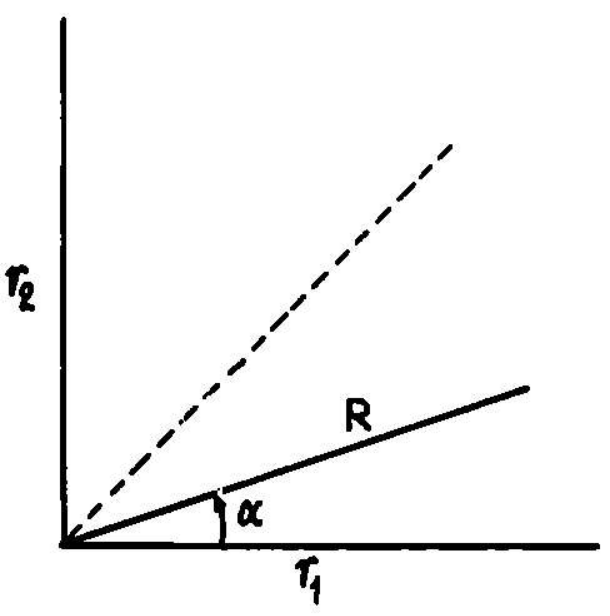

FIG. 1.--Connection of the hyperspherical coordinates $(R, \alpha)$ with the independent particle radial coordinates $\left(r_{1}, r_{2}\right)$.
$\Phi_{\ell_{1} \ell_{2} \mathrm{~m}}$ of Eq. 4 by eigenfunctions $\Phi_{\mu}$ of the operator in the square brackets of $\mathrm{Eq}$.

1. Each of these eigenfunctions is a superposition of $\Phi_{l_{1} l_{2} m}$ i however, the $\Phi_{\mu}$ corresponding to the lowest elgenvalues are found to consist mostly of a single $\Phi_{\ell_{1} \ell_{2}} \mathrm{~m}$ over a large range of $R$.

c) A post-adiabatic approximation is under development, which seeks to construct channel wavefunctions that are analogous to the adiabatic ones but yleld a weaker residual coupling - more nearly in line with experimental evidence - when superposed to construct a full solution of Eq. 1 . 
The following treatment is formulated for simplicity with reference to approximation a) but should apply in essence to the higher approximations. Note particularly the situation where one electron has reached much farther away from the nucleus than the other; it corresponds to large $\mathrm{R}$ and to values of $\alpha$ near 0 or $90^{\circ}$. The innermost of these electrons experiences then the full nuclear attraction, which implies that in $\mathrm{Eq} .4$

$$
\mathrm{g}^{\ell_{1} \ell_{2}} \mathrm{~m}(\mathrm{R} ; \alpha) \sim \begin{cases}\mathrm{f}_{\ell_{1}}\left(\mathrm{r}_{2}\right) \sim \mathrm{f}_{\ell_{1}}(\mathrm{R} \alpha), & \text { for } \alpha \ll 1, \\ \mathrm{f}_{\ell_{2}}\left(\mathrm{r}_{1}\right) \sim \mathrm{f}_{l_{2}}\left(\mathrm{R}\left[\frac{1}{2} \pi-\alpha\right]\right), & \text { for } \frac{1}{2} \pi-\alpha \ll 1 .\end{cases}
$$

where $\mathrm{f}_{\ell}$ is the radial wavefunction of an electron in the Coulomb field of a charge $Z$, regular at $r=0$.

With reference to our goal of extending the QDT, from single to double particle escape, recall from Sec. I that in the single-particle case essential information is provided by the connection between the behavior of radial hydrogenic wavefunctions near to and very far from the ionic core. Analogous information is provided in the two-particle case by the connection between the behavior of the radial function $F_{E}(R)$ at small $R$, where both electrons approach the core, and at large $R_{i}$ the weaker dependence on $R$ of the functions $\mathrm{g}^{\ell_{1} \ell_{2}} \mathrm{~m}$ 1s also relevant. However, a large value of $\mathrm{R}$ does nor ensure that both electrons are far from the core; e.g., the distance $r_{2}=R \sin \alpha$ of one electron from the nucleus may be small at large $R$ if $\alpha$ is sufficiently small. This circumstance will guide our further analysis.

We should also mention here the main current limitation to our knowledge of the solutions of the two-electron Eq. 1, even though it has little bearing on our immediate goal. The quasi-separability of variables embodied in Eqs. 3 and 4 actually holds only for small values of $\mathrm{m}$ or anyhow when either $R$ is not too large (say, $R \lesssim 10 \AA$ ) or $\alpha$ is $\ll 1$ (or $\sim \frac{1}{2} \pi$ ). A rather different separation obtains in the "Wannier region" of large $R$ and intermediate $\alpha\left(\sim 45^{\circ}\right)$ which leads to simultaneous high excitation or ionization of both electrons. The connection between the solutions of Eq. 1 in these two regions 
remains to be developed; its development will be required before one can apply the QDT to high double excitations or ionizations. However, the formulation of boundary conditions near the core can proceed at this time because it involves only the properties of wavefunctions at moderate values of $R$ or in the extreme ranges of $\alpha$.

\section{Boundary Conditions for a Pair of Electrons}

Consider now an ionic core with net charge $z$ from which two electrons have emerged into the surrounding space. Corsider also a spherical surface, with its center at the center of charge of the ion, and of radius $r_{0}$ sufficiently large for us to disregard: a) the occurrence of any core electron at distances $r>r_{0}$, and b) any correlation between electrons at $r>r_{0}$ and core electrons. (The experience of Ref. $4 \mathrm{~b}$ indicates that values of $r_{0}$ of the order of $5 \AA$ should meet these requirements for our purposes, except for large molecules.) Consider finally a pair of electrons in the space surrounding the core, at distances $\left(r_{1}, r_{2}\right)$ from the core's center both of which are larger than $r_{0}$. We represent the joint position $\left(\vec{r}_{1}, \vec{r}_{2}\right)$ of this electron pair by one point in 6 -dimensional space, and the pair of radial distance $\left(r_{1}, r_{2}\right)$ by a point in the section of that space shown in the diagram of Fig. 2. The locus of joint positions with $\left(r_{1}, r_{2}\right)>r_{0}$ consists of the non-hatched portion of the diagram. Throughout this portion, the Schroedinger equation of the electron pair is then represented by Eqs. 1 and 2, with $Z=z$, except for a reservation noted below.

To solve Eq. 1 in its limited range of validity, boundary conditions will have to be furmulated on the edge of this range, indicated in Fig. 2 by the dotdashed lines. The remark conceming Eq. 5 provides a basis for this purpose for the large-R portion of this edge, where $r_{0} \ll R$ and either $\alpha$ or $\frac{1}{2} \pi-\alpha$ is $\ll 1$. This portion of the edge consists of points of the configuration space at which the innermost of the two electrons lies on the surface surrounding the core, while the other is much farther away. On and near this boundary, one electron is subject, in essence, only to the unscreened attraction of the Ionic core with charge $z$, just as one electron is subject to the full nuclear charge $\mathrm{Z}$ at points near the coordinate axes of Fig. 1. The varlation of the 
joint wavefunction in the direction orthogonal to the boundary is then proportional to that of a wavefunction of a single electron in the Coulomb fleld, as Lin's wavefunction (Eq. 3) is proportional to the Coulomb function (Eq. 5) in the corresponding range of varlables. In our case, however, the presence of the ionic core in the hatched region of Fig. 2 causes the relevant Coulomb function to be phase-shifted, in general, instead of taking the form which is regular at the rigin. Thus we are led to a first application of quantum defect theory to our problem, in the restricted context of the interaction of a single electron with the core. The relevant phase shift is that which would be observed in the scattering of a single electron by the core, in a partial-wave state with the appropriate orbital momentum. These considerations are formulated by stating that, in the presence of an ionic core of effective radius, $r_{0}, E q .5$ is replaced by

$$
g^{\ell_{1} \ell_{2}} \mathrm{~m}(R ; \alpha) \sim \begin{cases}\mathrm{f}_{\ell_{1}}(R \alpha) \cos \pi \mu^{\prime} \ell_{1}-g_{\ell_{1}}(R \alpha) \sin \pi \mu^{\prime} \ell_{1}^{\prime} & \text { for } r_{2} \sim r_{0}, \alpha \ll 1, \\ f_{\ell_{2}}\left(R\left[\frac{1}{2} \pi-\alpha\right]\right) \cos \pi \mu^{\prime}{ }_{\ell_{2}}-g_{l_{2}}\left(R\left[\frac{1}{2} \pi-\alpha\right]\right) \sin \pi \mu_{\ell_{2}^{\prime}}^{\prime}, & \text { for } r_{1} \sim r_{2}, \frac{1}{2} \pi-\alpha \ll 1\end{cases}
$$

Here $g_{\ell}(r)$ indicates an irregular Coulomb wavefunction lagging in phase by $90^{\circ}$ with respect to $f_{\ell}(r)$. The phase shift is expressed as $\pi \mu^{\prime}$, in terms of a quantum defect, $\mu^{\prime}$, which is primed to stress that it pertains to the effect of the core on a single electron.

Note that Eq. 6 - as well as Eq. 5 before $1 t$ - contains two separate approximations, both of which hold in the limit of large $R$ and $\alpha \ll 1$ (or $\frac{1}{2} \pi-\alpha \ll 1$ ): a) When the distance of the outermost electron (1.e., the coordinate, $r_{1}$, in the lower part of Fig. 2 or of Fig. 1) is sufficiently large, the interaction of the second electron with the ionic core becomes independent of $r_{1}$. The effect of this interaction on the two-electron wavefunction outside the core is then represented by imposing a new value of its logarithmic derivative with respect to $r_{2}$, evaluated at $r_{2}=r_{0}, 1$.e., on the dot-dashed lines of Fig. 2 parallel to the $r_{1}$ axis. b) Application of the procedure of Sec. II requires, however, a boundary value of the derivative with respect to $\alpha$ taken at constant 


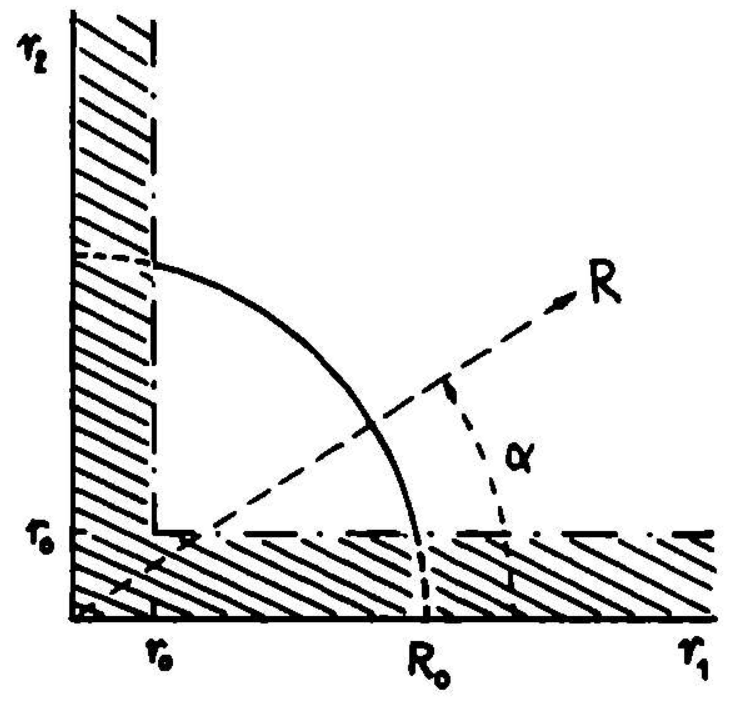

FIG. 2.--Diagram of boundary in configuration space. for Lin's function $g^{l_{1} l_{2}} \mathrm{~m}(R ; \alpha)$ new boundary conditions at $\alpha=\left(0, \frac{1}{2} \pi\right)$ which replace the corresponding condition of Sec. II, namely $\mathrm{g}_{\mathrm{m}}^{\ell_{1} \ell_{2}}=0$ on these boundaries. On this new basis one may return to Lin's procedure and calculate $\mathrm{g}^{\ell_{1} l_{2}} \mathrm{~m}(\mathrm{R} ; \alpha)$ throughout the range $0 \leq \alpha \leq \frac{1}{2} \pi$ by solving the approprlate wave equation derived from Eq. 1. ${ }^{6}$ [Altematuvely, one might solve the corresponding problem in one of the higher approximations indicated in Sec. II by b) or c).] Each elgenvalue of that wave equation is then entered as a potential term in the wave equation which is to determine the remaining radial factor $F_{E}(R)$ of the wavefunction (Eq. 3).

This second problem faces us now with the need for introducing a second boundary condition, this time on the low-R end of the range of integration in $R$. The treatment of Sec. II, for a point-like lonic core, Implies as a matter of course that $F_{E}(R)$ is the solution of the radial wave equation which is regular at $R=0$. [In fact, the presence of the factor $R^{\frac{5}{2}} \sin \alpha \cos \alpha$ on the left of Eq. 3 requires $F_{E}(R)$ to vanish at $R=0$, and $g^{l_{1} l_{2}}$ to vanish at $\alpha=\left(0, \frac{1}{2} \pi\right)$.] In our present problem, integration of the radial equation cannot extend to $R=0$, but must stop at some minimum radius $R=R_{0}$ at which the earlier step, namely, the establishment of Eq. 6 for large R, breaks down. This clrcumstance leads us to solve Eq. 1 only within a somewhat more restricted range of varlables, 
namely, within the non-latched ragion of Fig. 2 outside the circular arc with radius $R_{0}$. In this outer region we no longer select a particular solution $F_{E}(R)$ of the radial wave equation by requiring it to be regular at $R=0$; we consider instead a general solution represented by a superposition of two base solutions, $F_{E}$ and $G_{E^{\prime}}$ out of phase by $90^{\circ}$. The particular solution relevant to any one application will be determined by appropriate boundary conditions.

Considerations bearing on the choice of the radius $R_{0}$ have been mentioned above. Consider also that the exteinal electrons should become increasingly correlated with those of the core when both of their radial coordinates $\left(r_{1}, r_{2}\right)$ approach $r_{0}$ simultaneously. Accordingly, we shouldn't have txpected a solution of Eq. 1 to be applicable to our problem in the lower lefthand comer of the non-hatched region of Fig. 2. Thus, it appears approprlate on various grounds to exclude the sector of the diagram between this come: and the circle of radius $R_{0}$. The final selection of an appropriate value for $R_{0}$ should emerge from a numerical trial-and-error study of specific examples.

The whole sector of circle $R \leq R_{0}$, including its hatched portion, will then be regarded as the locus of appreciable correlation between the pair of emerging electrons and the ionic core. Within this finite region, an ab initio procedure should solve the Schroedinger equation for the complete system, 1.e. , core plus two electrons. This might be done according to Ref. 4 (a) for any arbitrary energy value $E$, seeking an eigenvalue, $b$, of the normal logarithmic derivative $2 \ln \Psi / \partial R$ on the boundary $R=R_{0}$. This boundary value need not be constant over the hyperspherical surface, $R=R_{0}$, but may vary in an approprate, preselected manner.

In the QDT treatment of a single emerging electron, its wavefunction is expanded over the boundary into spherical harmonics; for a pair of emerging electrons an analogous expansion will be into Lin's angular wavefunctions (Eq. 4), or into improved app:oximations thereof. [The orbital and spin momenta of the ionic core of the emerging electron(s) should actually be added to those; we disregard this matter here, assuming for simplicity that the core has zero angular momentum.] To each term of the wavefunction's expansion over the boundary surface, $R=R_{0}$ corresponds to a radial function that we indicate by 


$$
\mathrm{F}_{\mathrm{E}}^{\ell_{1} \ell^{2} \mathrm{~m}}(\mathrm{R}) \cos \pi \mu "-\mathrm{G}_{\mathrm{E}}^{\ell_{1} 2_{2} \mathrm{~m}} \text { (R) } \sin \pi \mu "
$$

where $\mu$ " is a second, radial, quantum defect. The complete wavefunction of the electron pair on and near this boundary will then be represented by a superposition of Lin's functions (Eq. 3),

$$
\begin{aligned}
& R^{\frac{5}{2}} \sin \alpha \cos \alpha \Psi
\end{aligned}
$$

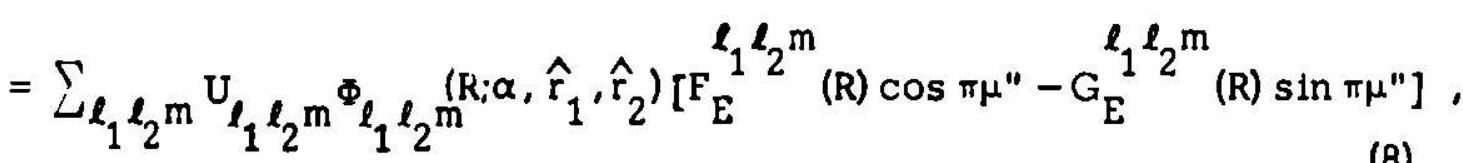

with the same "good" quantum numbers $\left(\mathrm{L}, \mathrm{M}, \mathrm{S}, \mathrm{M}_{\mathrm{S}}\right.$, and parity).

An $a b$ initio calculation of the wavefunction of the complete system "core +2 electrons" throughout the inner region $R \leq R_{0}$ may now be conducted along the line of Ref. $4(\mathrm{~b})$. The wavefunction is to be determined variationally for any given value of the energy $E$, with the boundary condition of foining smoothly to Eq. 8 at $R=R_{0}$ and treating the new quantum defect $\mu$ " as the eigenvalue to be maximized. The set of coefficients, $\mathrm{U}_{\ell_{1} \ell_{2} \mathrm{~m}^{\prime}}$ represents the corresponding eigenvector of the variational problem. The wavefunction expansion ( $\mathrm{Eq}$. 8), with the parameters so fitted, is intended to hold all the way to $R=\infty$; it should be understood, however, that the expansion index $\left(\ell_{1} \ell_{2} \mathrm{~m}\right)$ servas in Eq. 8 as a label for solutions of Eq. 1 , which constitute higher approximations than Eq. 3 , and which include effects of channel coupling that need not be discussed here.

Wavefunctions, such as Eq. 8, identified by an eigenvalus problem on a core's effective boundary, do not generally remain finite at $R=\infty$. According to $\mathrm{QDT},{ }^{2}$ the conditions at $\mathrm{R}=\infty$ are 1 mposed in a later stage of 1 ts procedure which need rot concem us here; they are met by constructing a superposition of all the degenerate wavefunctions ( $E q .8)$ with alternative eigenvalues $\mu^{\prime \prime}$ and eigenvectors $U_{\ell_{1} \ell_{2} m}$. The QDT also affords the altemative semi-empirical course of fitting experimental data (e.g., scattering amplitudes, energies of autolonizing levels) so as to determine the elgenquantum defects $\mu^{\prime \prime}$ and the corresponding elgenvectors $\mathrm{U}_{\ell_{1} \ell_{2} \mathrm{~m}}$ without solving the Schroedinger equation for $R \leq R_{0}$. However, in our problem of two-electron escape, one must still 
construct numerical solution of Eq. 1 in the outer region, 1.e., sets of wavefunctions $\Phi_{\ell_{1} \ell_{2} m}, F_{E}^{\ell_{1} \ell_{2} m}$, and $G_{E}^{\ell_{1} \ell_{2} m}$, utilizing semi-empirical quantum defects $\mu$ drawn from a separate study of the smaller system "core +1 electron."

Note the recursive aspect of the procedure outlined in this note. The boundary of the core region is separated into two sections, 1dentified in Fig. 2 by a dot-dashed and by a full line, respectively. The boundary condition on the first section depends on the interaction of a single electron with the 1onic core, it is represented by a first quantum defect, $\mu^{\prime}$, and it provides the start for integrating the wave equation 1 over one of its variables, namely, $\alpha$. The boundary condition on the second section depends on the interaction of two electrons with the core, it is represented by a second quantum defect, $1 \mathrm{\mu}$ ", and it provides the start for integrating the wave equation over a second rariable, namely, R. One would then surmise this sequence of operations to prove applicable to processes with successively higher numbers of electrons outside the core.

\section{Negative Ions}

Double excitation of a negative ion or excitation of a neutral atom (or molecule) by a slow electron yield a negatively charged complex consisting of a singly charged positive ion core with two electrons in the surrounding space. The simplest complex of this class, $\mathrm{H}^{-\star \star}$, whose ionic core consists of a bare nucleus as it does in the isoelectronic complex, $\mathrm{He}^{\star *}$, has been treated recently by the same method as $\mathrm{He}^{\star *} \cdot{ }^{6.9}$ The present paper is intended to apply to negatively charged complexes other than $\mathrm{H}^{-}$, whose ionic cores include electrons.

The relevant adaptations are substantially the same as have been required to adapt the QDT approach to the study of simple photodetachment. 2,10 Whereas in a singly excited neutral atom the excited electron is subject to a Coulomb fleld and its wavefunction is hydrogenic, an electron emerging from a neutral core is free and its radial wavefinction is a solution of the RiccatiBessel equation. Here again, as in the Coulomb probleni, the approprate solution depends on the short-range electron-core interaction, in a manner which 
1s represented by the coefficlents of the superposition of a standard pair of solutions, one regular and onr irregular at $r=0$. These coeificients are usually represented in terms of a phase shift, or of a scattenng length at very low energies, either of which serves the same capacity as the quantum defect. does in single-electron QDT. (In both cases of an Ionic or neutral core, the effect of core polarizability is understood to be included in the quantum defect or phase shift, but this procedure becomes less effective in the neutral case, where the polarization field is not overshadowed by a Coulomb field; we return to this point: below.)

Similarly, in a doubly-excited complex, the portion of the diagram of Fig. 2 , where $\alpha \ll 1$ and $R$ is large, pertains to a situation where one electron is much farther than the other from the core, and is fully screened by 1t. Here the radial function $F_{E} \quad(R)$ of $\mathrm{Eg}_{1}{ }^{3}$ represents the radial motion of the outermost electron. In this limit, $F_{E}^{l_{1} l_{2} m}(R)$ approaches then a hydrogenic wavefunction in the case of a neutral (or positively charged) complex. For a negative complex, it approaches, instead, a Rlccati-Bessel function. On the other hand, the innermost one of the two excited electrons is subject, in the same region, to the unscreened attraction of the lonic core; hence, the functions $f(R \alpha)$ and $g(R \alpha)$ of $E_{q} .6$ will be hydrogenic, even for a complex with a single negative charge. With these understandings, the treatment of Sec. III remains unchanged.

The polarizability of the Ionic core, which is generally disregarded in QDT applications, becomes more important in the case of negative complexes because of the absence of a long-range Coulomb fleld. This difficulty has hampered particularly a recent application to photodetachment from $\mathrm{Cs}^{-}$with simultaneous excitation of the core to its $6 \mathrm{p}$ levels. ${ }^{11}$ Since the $6 \mathrm{p}$ orbital does not extend radially much farther than the $6 \mathrm{~s}$ orbitals of the Cs ground state, 1.e., s snce we deal here with an intracore excitation, it seemed approprlate to regard the $6 \mathrm{p}$ electron as part of the neutral core and to treat the electron escape by the ordinary single-electron QDT. The Importance of core polarization suggests, however, that one might look upon the $\mathrm{Cs}^{-}$photodetachment as a two-electron process, in which both electrons in the valence shell 
of $\mathrm{Cs}^{-}$are treated equally by the approach of Sec. III, as moving in the field of a $\mathrm{Cs}^{+}$core.

\section{References}

1. M. I. Seaton, Proc. Phys. Soc. (London) 88,801 (1966).

2. U. tano, J. Opt. Soc. Amer. 65,975 (1975) and references therein.

3. P. G. Burke and W. D. Robb, Adv. Atom. and Molec. Phys. 11, 143 (1975).

4. (a) U. Fano and C. M. Lee, Phys. Rev. Lett. 31,1573 (1973).

(b) ( M. Lee, Phys. Rev. A 10, 584 (1974).

5. U. Fano, Atomic Physics, Vol. 1 (Plenum, New York, 1969), p. 209 and references therein.

6. U. Fano and C. D. Lin, Atomic Physics, Vol. 4 (Plenum, New York, 1975), p. 47; C. D. Lin, Pinys. Rev. A 10,1986 (1974).

7. J. H. Macek, J. Phys. B 2, 831 (1968).

8. The discussion given here implies for simplicity that the ionic core has zero anglilar momentum, whereby the electron-core interaction cannot change the orbital momentum $\ell_{1}$ ( or $\ell_{2}$ ) of the excited electron. This restriction should be removed by applying the multichannel QDT, which interrelates the boundary values of $\mathrm{g}^{\ell_{1} \ell_{2}} \mathrm{~m}(\mathrm{R} ; \alpha)$ functions with different orbit numbers $\left(l_{1} l_{2}\right)$.

9. C. D. Lin, Phys. Rev. A 12, 493 (1975); Phys. Rev. Lett. 35, 1150 (1975).

10. A. R. P. Rau and U. Fano, Phys. Rev. A 4, 1751 (1971).

11. C. M. Lee, Phys. Rev. A 11,1592 (1975). 